\title{
Repositioning of a Novel GABA-B Receptor Agonist, AZD3355 (Lesogaberan), for the Treatment of Non-alcoholic Steatohepatitis
}

\author{
Dipankar Bhattacharya \\ Icahn School of Medicine at Mount Sinai, New York, \\ Christine Becker \\ Icahn School of Medicine at Mount Sinai, New York, \\ Benjamin Readhead \\ Icahn School of Medicine at Mount Sinai, New York, \\ Nicolas Goossens \\ Icahn School of Medicine at Mount Sinai, New York, \\ Jacqueline Novik \\ Icahn School of Medicine at Mount Sinai, New York, \\ Maria Isabel Fiel \\ Icahn School of Medicine at Mount Sinai, New York, \\ Leslie P. Cousens \\ Emerging Innovations, Discovery Sciences, R\&D, AstraZeneca, Boston, MA, \\ Björn Magnusson \\ Discovery Biology, Discovery Sciences, R\&D, AstraZeneca, Gothenburg \\ Anna Backmark \\ Discovery Biology, Discovery Sciences, R\&D, AstraZeneca, Gothenburg \\ Ryan Hicks \\ BioPharmaceuticals R\&D Cell Therapy, Research and Early Development, Cardiod Metabolism (CVRM), \\ BioPharmaceuticals R\&D, AstraZeneca, Gothenburg \\ Joel T. Dudley \\ Icahn School of Medicine at Mount Sinai, New York, \\ Scott L. Friedman ( $\nabla$ scott.friedman@mssm.edu ) \\ Icahn School of Medicine at Mount Sinai, New York,
}

\section{Research Article}

Keywords: Fibrosis, NAFLD, hepatic stellate cell, drug repurposing, informatics, therapeutics

Posted Date: April 12th, 2021

DOI: https://doi.org/10.21203/rs.3.rs-386482/v1

License: (c) This work is licensed under a Creative Commons Attribution 4.0 International License. Read Full License 


\section{Abstract}

Non-alcoholic steatohepatitis (NASH) is a rising health challenge, with no approved drugs. We used a computational drug repositioning strategy to uncover a novel therapy for NASH, identifying a GABA-B receptor agonist, AZD3355 (lesogaberan) previously evaluated as a therapy for esophageal reflux. AZD3355's potential efficacy in NASH was tested in human stellate cells, human precision cut liver slices (hPCLS), and in vivo in a well-validated murine model of NASH. In human stellate cells AZD3355 significantly downregulated profibrotic gene and protein expression. Transcriptomic analysis of these responses identified key regulatory nodes impacted by AZD3355, including Myc, as well as MAP and ERK kinases. In PCLS, AZD3355 down-regulated collagen $1 \mathrm{a} 1$, aSMA and TNF-a mRNA as well as secreted collagen1a1. In vivo, the drug significantly improved histology, profibrogenic gene expression, and tumor development in a robust murine model of NASH, which was comparable to activity of obeticholic acid, an advanced investigational therapy for this disease. These data identify a well-tolerated clinical stage asset as a novel therapy for human NASH through its hepatoprotective, anti-inflammatory and antifibrotic mechanisms of action. The approach validates computational methods to identify novel therapies in NASH in uncovering new pathways of disease development that can be rapidly translated into clinical trials.

\section{Introduction}

Nonalcoholic steatohepatitis (NASH) is a serious and escalating health threat in the United States, affecting between 6.5 million to 16.3 million Americans ${ }^{1}$, with a rising incidence paralleling the obesity epidemic. NASH represents a more advanced form of non-alcoholic fatty liver disease (NAFLD), which affects up to 60 million Americans and hundreds of millions worldwide ${ }^{2,3}$. NASH disease is characterized by hepatocellular fat, inflammation and injury with accumulation of fibrillar collagen generated by activated hepatic stellate cells, the principle fibrogenic cell in liver ${ }^{4}$. NASH can lead to liver failure and hepatocellular carcinoma $(\mathrm{HCC})^{5}$, and will supplant hepatitis $\mathrm{C}$ as the primary indication for liver transplantation in the coming years, along with alcoholic liver disease, which has many overlapping pathogenic pathways with NASH ${ }^{6}$.

The expanding public health impact of NASH underscores the urgent need to develop novel therapies that prevent progression and/or reverse fibrosis to improve outcomes; however, there are no approved therapies to date. An incomplete understanding of molecular mechanisms underlying NASH is a major impediment to therapeutic development. Further, a number of immune, metabolic, fibrotic, and other major pathways contribute to NASH pathogenesis ${ }^{4}$ adding to the complexity of identifying target-based therapeutic mechanisms. Obeticholic acid (OCA or INT-747 or 6alphaethylchenodeoxycholic acid (6-ECDCA)) is the most advanced investigational drug for NASH thus far, but is not yet FDAapproved due to safety concerns. Moreover, this agent improves NASH fibrosis in only $23.1 \%$ of patients in a phase 3 trial, compared to $11.9 \%$ of placebo-treated patients ${ }^{7}$.

In drug development only 6-7\% of drugs that successfully enter Phase I trials advance to FDA approval ${ }^{8,9}$. The major reasons for drug failures include adverse effects (toxicity), inadequate efficacy, off-target effects and poor pharmacokinetic (DMPK) profiling ${ }^{10-12}$.

More importantly, many drugs that have clean safety profiles are shelved because of insufficient efficacy or commercial decisions related to the available market and competitive landscape. Some of these agents can be repurposed for treating diseases unrelated to their original clinical indication based on an emerging understanding of pathobiology, and enhanced by computational tools to search vast databases of publicly available knowledge ${ }^{13,14}$. These approaches identify connections between drug action and disease that may not be intuitive to even the most knowledgeable physician-scientist. Repurposing can have major advantages in time-and cost-efficiency. For example, Phase 1 safety studies of the original clinical development program can be utilized to assess the risk-benefit ratio a newly proposed indication, and where deemed appropriate, accelerate into Phase 2 testing of efficacy to significantly reduce development timelines and costs ${ }^{15}$. In addition, drug repurposing reduces risk of failure from toxicity and can lower the cost of drug development since these FDAapproved drugs have passed regulatory scrutiny and sometimes post-market surveillance as well. 
Traditional drug discovery relies on target-based or phenotypic screening, with further development largely driven by clinical observations and pharmacology. Recent evidence suggests that powerful bioinformatic methods applied to systematically mine the wealth of available genomic, molecular, and clinical data can identify additional connections between disease and drug signatures that are not mechanistically intuitive, but that implicate novel indications and/or disease subsets. The potential to develop repurposed drugs to treat NASH has been buoyed by the development of analytic methods to mine transcriptomic data for evidence of a therapeutic signal ${ }^{16}$. Mining large volumes of genomic, molecular, and clinical data offers new approaches for the generation of novel, testable hypotheses connecting drug activity with unmet clinical needs. High-dimensional molecular data, such as transcriptomic profiling, are routinely used to generate rich perspectives of biological states of interest and offer exciting opportunities to exploit computational methods to better understand human response to disease and treatment. A powerful example of this is computational drug repositioning, which leverages systematic approaches to characterize and relate disease and drug-induced states to identify novel indications for existing therapies, thus prioritizing valuable experimental validation efforts. The aim of computational drug repurposing is the more rapid and systematic identification of novel therapeutic strategies compared to traditional modes of drug development.

In the current study we used a computational drug repurposing strategy to identify an FDA-approved gamma-aminobutyric acid receptor $B(G A B A-B)$ agonist as a potential new therapy for NASH. We then explored the potential efficacy of AZD3355 (Lesogaberan) ${ }^{17,18}$, an agent that previously failed in a Phase $2 b$ human trial fortreatment of gastroesophageal reflux disease (GERD) ${ }^{19}$, as a novel treatment for NASH using a combination of culture and in vivo studies.

\section{Results}

\section{Computational drug repurposing identifies AZD3355 as a potential treatment for NASH}

Using a connectivity mapping approach, we generated RNA-sequence profiles on a defined set of 32 clinic-ready compounds, under 4 conditions (e.g. "Compound-A A549 High dose”, “Compound-A MCF7 High dose”, "Compound-A A549 Low dose", "Compound-A MCF7 Low dose"), and compared these to cell-matched DMSO-treated control samples to define a differential gene expression signature for each (Figure 1A). Each compound-condition was then compared to a disease gene expression library (generated from publicly available data ${ }^{20,21}$ ) and a connectivity score was obtained for each compound-condition/disease pair (Figure 1B).

Given the high unmet need to develop novel therapeutic strategies for improved treatment of NASH, we were interested to note significant inverse connections (FDR <0.01) between all four AZD3355 signatures and a NASH gene expression signature (GSE24807). Of the 32 compounds profiled within our study, only AZD3355 demonstrated this concordant inverse connectivity across all four experimental conditions (Figure 1C-D).

To further evaluate whether the transcriptomic relationship between AZD3355 and the NASH gene expression signature was unusually strong, we performed the same repurposing analysis for all 1,309 compounds (including many FDA approved compounds) in Connectivity Map (a collection of genome-wide transcriptional expression data from cultured human cells treated with bioactive small molecules) against the NASH signature and found that the connectivity score for AZD3355 was in the top $2 \%$ of predictions for NASH when ranked among all 1,309 compounds. Therefore, we regarded the connection between AZD3355 and NASH to be significant, even in the context of a large set of compounds with diverse mechanisms and disease indications.

To further investigate the context of AZD3355's effects upon NASH biology, we tested the AZD3355 gene signatures against additional human NAFLD liver and NAFLD therapeutic transcriptomic datasets (Figure 1E). We observed consistent inverse expression of gene signatures between AZD3355 and human NASH. Furthermore, we found enrichment of the AZD3355 gene signatures in hepatic transcriptomic datasets of therapeutic interventions known to improve NAFLD (bariatric surgery and rosiglitazone therapy). These findings suggest that AZD3355 induces a gene signature similar to candidate therapies 
of NASH and opposite to human NASH, thus establishing the rationale to directly test AZD3355's activity in models of fibrosis and NASH in culture and in vivo.

\section{AZD3355 attenuates hepatic stellate cell activation}

We assessed the effects of AZD3355 on hepatic stellate cell gene expression in both LX-2 cells, an immortalized human stellate cell line 22 and primary human hepatic stellate cells (phHSCs) to assess the drugs impact on fibrogenesis. Cells were treated with $30 \mathrm{nM}$ and $100 \mathrm{nM}$ AZD3355 for 48 and 72 hours. Both LX-2 and phHSCs were culture-activated through growth on uncoated plastic in the presence of serum, and expressed high level of key profibrotic genes in the absence of additional stimuli. DMSO and the multi-kinase inhibitor sorafenib $(7,500 \mathrm{nM})$ were used as vehicle and as a positive control, respectively. In LX-2 cells, there was down-regulation of profibrotic genes by AZD3355 (Figure 2A, solid bars). To exclude toxicity as a reason for reduced gene expression, fresh media without drug was restored to the cells after 48 and 72 hours, respectively, and 72 hours after that mRNA expression resumed baseline levels (Figure 2A, striped bars).

In parallel, we evaluated the effects of AZD3355 on phHSCs. The cells were incubated with $30 \mathrm{nM}$ or $100 \mathrm{nM}$ AZD3355 48 and 72 hours, compared to vehicle. Gene expression for COL 1a1, aSMA, $\beta$-PDGFR, TGF $\beta$-R1, TIMP1 and MMP2 was significantly down-regulated by $30 \mathrm{nM}$ (Figure 2B). Selection of optimal effective concentrations of AZD3355 was determined by cytotoxicity, proliferation and apoptosis assays; both LX-2 and phHSCs tolerated up to 100 nM AZD3355 for 72 hours (Suppl Figure S2A and B).

\section{Transcriptomic / pathway analysis in phHSCs in response to AZD3355}

Because fibrogenic gene expression in phHSCs was significantly down-regulated by $30 \mathrm{nM}$ of AZD3355 at 72 hours (Figure 2B), we selected this condition to perform transcriptomic analysis in order to more broadly characterize genes regulated by AZD3355. Twenty two genes were differentially expressed (P-adj < 0.1) between AZD3355 treatment and vehicle groups (Figure 3A). Included in the up-regulated genes are STAR-related lipid transfer domain containing 9 (STARD9); sterol regulatory element binding transcription factor 1 (SREBF1); and Kielin cysteine rich BMP Regulator (KCP). Cancer-related transcripts of AKR1C2, TPT1 and RPS27A were also down-regulated by AZD3355 in phHSCs (Figure 3A). We evaluated differential enrichment to establish if there were biological pathways enriched within this gene list. The top pathways identified were 'metabolism of steroids', 'activation of gene expression by SREBF' and 'activation of gene expression by SREBP'(Figure 3B).; the findings are interesting given the stellate cell's important role in storing lipids within retinyl esters ${ }^{23}$, but were not further pursued.

We next identified the cell-signaling networks associated with these genes using the computational tool eXpression2Kinases (X2K) ${ }^{24}$. Transcription factor enrichment analysis revealed MYC as the top predicted transcription factor most likely regulating the observed changes in mRNA expression (Suppl Figure S3A). The protein-protein interaction expansion analysis using the Genes2Networks algorithm identified the sub-network of connected transcription factors that regulated these genes (Suppl Fig S3B). Kinase enrichment analysis prioritized the protein kinases known to phosphorylate substrates within the subnetwork of transcription factors and the intermediate proteins that connect them. MAP and ERK kinases were among the top kinases identified (Suppl Fig S3C). Together this data was used to generate the network (Suppl Fig S3D).

\section{AZD3355 inhibits COL1A1, aSMA, TNFa mRNAs and secreted Col1A1 in diseased human liver slices}

To assess the effects of AZD3355 on pro-fibrotic and inflammatory response genes in a more physiological context, we used the precision cut human liver slice model (PCLS), which preserves all cell types within their native architecture to uniquely capture endogenous cell-cell interactions ${ }^{25}$. PCLS from de-identified resection samples of injured human liver were used to test the activity of AZD3355 (250 nM and $500 \mathrm{nM}$ ) or DMSO (negative control) following 24 hour incubation. Three liver samples were used, 1 male and 2 female, with ages ranging from 60 to 74 years with different etiologies, including one 
NASH liver (Table 1). After 24 hours, there was dose-dependent, significant down-regulation of key fibrogenic genes COL 1A1 and aSMA as well as the inflammatory response gene TNFa by AZD3355 in all three patients liver slices (Figure 4A). ELISA was used to examine the effect on secreted Col1a1 of AZD3355. There was significant reduction of secreted Col1a1 by AZD3355 treatment compared to vehicle in all three slice donors consistent with effects on of COL 1A1 mRNA (Figure 4B). There was no cytotoxicity from AZD3355 based on lack of LDH release in the culture media (Suppl Figure S4).

\section{Effect of AZD3355 in a murine NASH model in vivo}

We have previously established a murine model that closely resembles human NASH following 12 or 24 weeks of a high fat, high fructose, high cholesterol ('Western') diet with low dose $\mathrm{CCl}_{4}$ administration $\mathrm{IP}^{26}$. The model faithfully replicates both the progression of disease histologically from fibrosis to cancer, the transcriptomic features and metagenome of human NASH ${ }^{27}$; we refer to this model as the FAT-NASH (Fibrosis And Tumors, NASH) model. In the FAT-NASH model, AZD3355 (10 $\mathrm{mg} / \mathrm{kg}$ or $30 \mathrm{mg} / \mathrm{kg}$ ) was administered by gavage twice daily, 5 days per week, beginning at 13 week of the $\mathrm{CCl}_{4} / \mathrm{diet}$ protocol and continued for the remaining 12 weeks of the NASH protocol.

In mice administered AZD3355 compared to the vehicle group with NASH, the average body weight was significantly decreased by the $30 \mathrm{mg} / \mathrm{kg}$ dose, but not $10 \mathrm{mg} / \mathrm{kg}$, beginning in week 2 of treatment (week 14 of the study) in males, and from week 3 (week 15 of the study) in females (Suppl Figure S5A). The reduced body weight was not due to the toxic effect of AZD3355 dosing, as no significant differences in food or water intake were observed before and during AZD3355 administration (Suppl Fig S5B and S5C).

AZD3355 also dose-dependently reduced hepatomegaly in both male and female mice. At $30 \mathrm{mg} / \mathrm{kg}$ AZD3355, the average liver weight and liver-to-body weight ratio were significantly decreased compared to vehicle-treated animals (Suppl FigS6A and S6B). Necro-inflammatory activity was assessed in part by serum aspartate aminotransferase (AST) and alanine aminotransferase (ALT) levels. Both in male and female mice the average AST and ALT, and in female mice, average triglyceride levels were significantly reduced (Suppl Fig S6C and S6D). Average triglyceride trended towards reduction in male mice in response to AZD3355 compared to vehicle (Suppl Fig S6C).

The NAFLD activity score (NAS) was assessed blindly according to NASH-CRN criteria by an experienced liver pathologist

28. In male mice receiving vehicle, the NAS was $7.4 \pm 0.2$, which was reduced to $6.6 \pm 0.6$ in treated with $30 \mathrm{mg} / \mathrm{kg}$ AZD3355. In mice treated with $30 \mathrm{mg} / \mathrm{kg}$ OCA, NAS scores were reduced to $4.7 \pm 0.7$ in male and $4.7 \pm 0.6$ in female mice (Suppl Fig S7B and 7C, Table 2). $30 \mathrm{mg} / \mathrm{kg}$ AZD3355 treatment as well as OCA also significantly reduced fibrosis stages in both male and female FAT-NASH mice (Figure S7B and S7C)

\section{Antifibrotic activity of AZD3355 in murine NASH}

We also assessed the effect of AZD3355 on murine NASH by quantifying whole liver mRNA and protein expression for key markers of hepatic fibrogenesis. There was a dose-dependent down-regulation of mRNAs for Col1a1, aSma, and Pdgf-R $\beta$ in both male and female animals compared to vehicle (Figure 5A). Interestingly, gene expression for TGF $\beta$-R1, TIMP1 and MMP2 was down-regulated in female mice only (Figure 5A). In mice treated with OCA, Col1a1and aSma gene expression were down-regulated in both male and female mice, whereas $P d g f-R \beta$ was reduced in male mice; TIMP1, TIMP2 and MMP2 were reduced in female mice (Figure 5A).

We also measured expression of Col1a1 and aSma by Western Blot from whole liver lysates (Figure 5B and 5C). Both Col1a1 and aSMA proteins were reduced in male mice administered either 10 or $30 \mathrm{mg} / \mathrm{kg}$ AZD3355 (Figure 5C). In female mice, aSMA protein expression was reduced with both 10 and $30 \mathrm{mg} / \mathrm{kg}$ AZD3355, however Col1a1 protein expression was down-regulated in females treated with AZD3355 $30 \mathrm{mg} / \mathrm{kg}$ (Figure 5C). In OCA treated animals Col1a1 and aSMA protein were significantly down-regulated in both male and female mice (Figure $5 \mathrm{C}$ ).

\section{AZD3355 reduces collagen content in a murine NASH model}


To assess hepatic fibrosis in murine NASH, collagen accumulation was quantified by morphometry of picrosirius redstained liver sections from vehicle and drug-treated mice. Severe bridging fibrosis was present in both male and female mice administered vehicle only, which was reduced in AZD3355-treated animals (Figure 6A). By morphometry of Sirius Red stained tissue collagen content was significantly reduced in both male and female animals by 10 or $30 \mathrm{mg} / \mathrm{kg}$ AZD3355, (Figure 6B). This results are consistent with the pathological scoring of fibrosis (Figure S7B and S7C). Collagen deposition in obeticholic acid- treated control mice was also significantly reduced in both male and female groups (Figure 7B).

\section{Reduced hepatocellular carcinoma development in FAT NASH mice treated with AZD3355}

A consistent feature of this FAT-NASH model is the development of hepatocellular carcinoma (HCC) in all male animals at 24 weeks ${ }^{26}$. Similarly, in the current study there was robust tumor development with vascular invasion in some animals (Suppl Figure S8). In males, 100\% developed HCC in the vehicle-treated group. In females treated with vehicle, 50\% developed HCC (Figure 7A). AZD3355 treatment of male mice led to a dose-dependent reduction of tumor development; $11 \%$ and 22\% mice did not develop HCC by AZD3355 $10 \mathrm{mg} / \mathrm{kg}$ and $30 \mathrm{mg} / \mathrm{kg}$, respectively and the total number of tumors was significantly reduced (Figure 7A). In OCA treated animals the number of tumors was reduced in both male and female mice.

\section{Discussion}

In this study we have computationally identified AZD3355 as a potential therapeutic for NASH and have experimentally validated this in culture, ex vivo and using an in vivo model. AZD3355 was initially designed to be a potent, peripherallyrestricted gamma-aminobutyric acid receptor $B(G A B A-B)$ receptor agonist with a preclinical therapeutic window to inhibit transient lower esophageal sphincter relaxations (TSLERs) with over 40X greater potency compared to a standard therapy, Baclofen 29,30 . However, Phase $2 b$ testing indicated that AZD3355 did not achieve a clinically meaningful effect on GERD outcomes 19,31 , and further development in this indication was no longer pursued by AstraZeneca Pharmaceuticals.

Our data in these NASH-related studies demonstrate that the compound, a GABA-B receptor agonist, has antifibrotic, antiinflammatory, and hepatoprotective activity in cultured primary and immortalized human stellate cells, in human liver slices, and in a murine model of NASH whose features closely mirror human $\mathrm{NASH}$. These findings reinforce the promise of computational based drug repurposing to more rapidly identify therapeutic candidates, an approach that has been validated in other diseases such as inflammatory bowel disease ${ }^{14}$ and cancer ${ }^{13}$, but not previously in NASH. Thus, our findings add to a growing body of evidence validating the reliance on unbiased data representations of complex molecular networks that underly disease to uncover novel pathogenic pathways or therapeutic targets.

The direct reduction in fibrosis by AZD3355 in cultured primary and immortalized human HSCs and in vivo in mouse reveals a novel intracellular pathway of gene regulation in this cell type, the principal fibrogenic cell in liver injury following its activation into proliferative myofibroblasts ${ }^{32,33}$. The finding is consistent with an earlier study describing effects of GABA-B receptor agonism on fibrosis and aSMA expression (a classical marker of HSC activation) in cultured rat stellate cells, and in vivo in $\mathrm{CCl}_{4}$ injury in rats ${ }^{34}$. Interestingly, both that study and our own data confirm a direct antifibrotic activity but no effect of GABA-B agonism on stellate cell proliferation. GABA-B receptor agonism also inhibited TGFb1 secretion in the previous publication ${ }^{34}$, which could account for its antifibrotic effect in our studies as well; although we did not measure this cytokine directly, we documented reduced TGFb1 gene expression in vivo. Interestingly, we identify Myc as the top transcription factor regulated in cultured HSCs in response to AZD3355 which indicates it may play a central role in regulating the observed changes in mRNA expression; Myc which has previously been implicated in HSC activation ${ }^{35,36}$, but

has never been fully explored in this cell type. Since inactivating oncogenic myc is a major target of cancer therapies ${ }^{37}$, this finding raises the prospect of further defining the role of myc as a direct antifibrotic target in liver fibrosis as well.

Interestingly, AZD3355 reduced expression of several other liver HCC-related genes in HSCs, AKR1C2 ${ }^{38}, \mathrm{TPT}^{39}, \mathrm{RPS} 15 \mathrm{~A}^{40}$ and RPS27A ${ }^{41}$. While HSCs are not the cell of origin in HCC, reduced expression of these genes in response to AZD3355 
might alter their potential to promote tumors in NASH, as stromal gene expression can be an important determinant of HCC outcomes ${ }^{42,43}$.

Our findings also suggest anti-inflammatory and hepatoprotective activities of AZD3355 as well based on reduced TNF-a mRNA in human liver slices, and reduced serum AST and ALT in vivo in the NASH model, respectively. These effects may indirectly further reduce HSC activation and fibrogenesis, but more mechanistic studies are warranted to firmly establish this possibility. Nonetheless, the multiple direct and indirect benefits of AZD3355 reinforce its appeal as a potential therapy for NASH. To date, the most advanced NASH therapy in phase 3 trials is obeticholic acid, which like AZD3355, has multiple targets of activity in the disease $7,44,45$; the drug showed efficacy in a phase 3 trial ${ }^{7}$ but is not yet approved. Here we further establish the therapeutic effect of obeticholic acid as a positive control in our study, which has comparable activity to AZD3355. The rising number of drug failures in $\mathrm{NASH}^{46,47}$ by agents that have a single cellular or molecular target indicate that agents with multiple targets such as AZD3355 may be more promising.

The sex difference in the extent of injury following AZD3355 is an additional finding of interest, with lower AST/ALT in female mice associated with decreased a-SMA and collagen expression in vivo, as well as greater liver cancer development in males. While a greater propensity towards liver cancer in male mice and humans is well described and thought to reflect different regulatory pathways ${ }^{48}$, sex differences in human NASH have not been as well characterized ${ }^{49}$. Nonetheless, sex is a significant factor in determining patterns of gene expression in human $\mathrm{NASH}^{50,51}$, and further studies addressing gender as a determinant of NASH risk and disease in animal models are needed.

The heterogeneous responses to AZD3355 among human precision-cut liver slices from different donors is also a noteworthy observation, and may mirror the challenges of heterogeneity in clinical trials as well. While reliance on inbred mouse strains or disease modeling provides more interpretable readouts of drug efficacy, these models overestimate drug activity following their translation into human trials. This has been especially true in NASH, where in vivo modeling in rodents typically yield more optimistic results than is subsequently seen in human clinical trials. Thus, greater reliance on systems such as PCLS that reflect real-world heterogeneity may be highly informative in predicting clinical success, and could provide insights into the factors (i.e. genetic, epigenetic or even metagenomic) that contribute to this heterogeneity.

These results provide further validation of data-driven, hypothesis-free approaches to uncover novel therapies using existing drugs with established safety profiles ${ }^{16,52}$. In the example of AZD3355, the agent had no apparent link to fibrotic liver disease, yet transcriptomic profiling uncovered its potential efficacy, which we have validated in this study. Moreover, since the drug's mechanism of action includes potent direct antifibrotic activity towards fibrogenic cells, it may be active in other chronic as well as fibrotic diseases in liver and other tissues.

\section{Materials And Methods}

\section{Computational drug repurposing method to identify candidate NASH therapies:}

We used a modified connectivity mapping approach to identify potential novel use cases for a collection of 32 compounds, each of which had completed Phase 1 testing or greater (https://openinnovation.astrazeneca.com/datalibrary.html\#transcriptomicprofilingdata). Compound signatures were obtained by exposing cell lines (A549 and MCF7, from ATCC ${ }^{53}$ ) to each compound, at a high and low dose, and generating a RNASeq signature, thus resulting in four separate signatures for each compound (e.g. "Compound-1 A549 High dose”, "Compound-1 MCF7 High dose”, "Compound1 A549 Low dose”, “Compound-1 MCF7 Low dose”). These were then compared to a disease library (generated from publicly available data ${ }^{20,21}$ ) and a connectivity score was obtained for each compound-condition/disease pair. This approach has previously been described by us and others ${ }^{20,54}$. Compound-condition/disease connectivity scores, that were significantly (FDR < 0.01) negative (connectivity score $<0)$, indicate that under that particular cell-dosage combination, the compound is predicted to "normalize" the disease signature towards the transcriptomic profile observed in healthy controls. 
The NASH signature used in the disease mapping step was generated from liver biopsies collected from 12 NASH patients, and five control subjects ${ }^{55,56}$ available from Gene Expression Omnibus (Accession: GSE24807).

\section{Gene set enrichment analysis of AZD3355 against human NAFLD data sets}

We generated AZD3355 gene expression perturbation signatures with up- and down-regulated genes in the A549 and MCF7 cell lines with a false discovery rate cutoff of $p<0.05$. We then used these gene sets as inputs for a Gene Set Enrichment Analysis ${ }^{57}$ to quantify enrichments of these AZD3355 signatures in human NAFLD datasets available from Gene Expression Omnibus (see Figure 1E for references).

\section{Analysis of AZD3355 effects in immortalized (LX-2) and primary human stellate cells (phHSCs):}

AZD3355 (FW 141.08) was supplied by AstraZeneca Pharmaceuticals to purity > 99\% by HPLC. The development and structure of AZD3355 has been previously described ${ }^{58}$. For cell culture studies, AZD3355 was reconstituted in normal saline ( $0.9 \%$ sodium chloride) at $2 \mathrm{mM}$ concentration stock solution followed by a series of working concentrations of 1,3 , 10, 30, 100 and $300 \mathrm{nM}$ in DMEM cell culture medium (Thermo Fisher Scientific, MA), supplemented with 0.1\% BSA. Both stock and working solutions were made fresh before each experiment. As a positive control the cells were treated in parallel with sorafenib (LC laboratories, MA) at 7,500 nM concentration dissolved in sterile DMSO, since this drug has antifibrotic activity in cultured stellate cells ${ }^{59}$.

The activity of AZD3355 was analyzed in LX-2 cells, an immortalized human hepatic stellate cell line ${ }^{22}$ and primary human hepatic stellate cells (phHSCs) isolated from discarded remnants of surgically resected human livers ${ }^{60}$, which lacked patient identifiers. Use of these de-identified tissues to generate phHSCs was approved by the Institutional Review Board (IRB) at the Icahn School of Medicine at Mount Sinai, NY. Procedure of phHSCs isolation, culture and their purity assessment by immunostaining has been described previously ${ }^{61}$. Prior to all drug incubations, both LX-2 cells and phHSCs were maintained overnight in starvation media comprised of DMEM supplied with $0.1 \%$ BSA (without antibiotics) to synchronization cell metabolic activities. The cells were incubated with incremental concentrations of AZD3355 or sorafenib for 24,48 and 72 hours.

\section{Cytotoxicity assay:}

5,000 LX-2 cells or 10,000 phHSCs were plated per well in 96 well plates, serum-starved overnight, then incubated with either vehicle different concentrations of AZD3355 in starvation media for the indicated durations. MTS assays were performed using CellTiter 96 Aqueous One Solution Cell Proliferation Assay kit (Promega, WI) according to the manufacturer's protocol.

\section{Cell proliferation assay:}

LX-2 cells (5,000 cells per well) or phHSCs $(10,000$ cells per well) were plated in 96 well plates, serum-starved overnight, then incubated with incremental concentrations of AZD3355 in starvation media. After end of drug incubation (24, 48 or $72 \mathrm{~h})$, the cells were labeled with BrdU for either 2 hours (for LX-2 cells) or 16 hours (for phHSCs) at $37^{\circ} \mathrm{C}$ in $5 \% \mathrm{CO}_{2}$. Cell proliferation was quantified using the cell proliferation ELISA, BrdU colorimetric kit (Roche, NY) according to manufacturer's instructions. Absorbance was measured at $370 \mathrm{~nm}$ with reference wavelength at $492 \mathrm{~nm}$.

\section{Cell apoptosis assay:}

LX-2 cells (5,000 cells per well) or phHSCs (10,000 cells per well) were plated in 96 well clear bottom black plates. After overnight serum starvation the cells were incubated with AZD3355 at the indicated concentration or 3\% DMSO (as a positive control to induce apoptosis). After $72 \mathrm{hrs}$, the fluorescence signal of Caspase-3 and-7 activities were measured using a Synergy HT (BioTek Instrument Inc., VT) spectrofluorometer using Apo-ONE Homogeneous Caspase-3/7 Assay kit (Promega, WI) according to the manufacturer's protocol. 


\section{RT-Quantitative PCR in human HSCs:}

LX-2 (150,000 cells) or phHSCs (200,000 per well) were plated in 6-well plates. After overnight serum starvation the cells were incubated with either vehicle, AZD3355 or sorafenib at the indicated concentrations for 48 or 72 hours. For LX-2 cells the vehicle and the drug were replaced after 48 or 72 hours by normal DMEM with $10 \%$ FBS, then maintained for an additional 48 or 72 hours, respectively. Cells were harvested and total RNA was extracted using RNeasy Mini Kit (Qiagen, CA) according to manufacturer protocols. $0.5 \mu \mathrm{g}$ of total RNA was used for reverse transcription with 'RNA to cDNA EcoDry Premix (Double Primed) Kit' (Clontech, CA). Expression of fibrogenic genes was quantified by qPCR using custom designed primers (Sigma-Aldrich, MO) and iQ SYBR Green Supermix (Bio-Rad, CA) on a LightCycler 480 II (Roche Diagnostics Corporation, IN). Glyceraldehyde-3-phosphate dehydrogenase (GAPDH) were used as housekeeping gene to determine the relative expression of fibrogenic genes.

\section{RNA sequencing analysis of primary human hepatic stellate cells (phHSC):}

Two hundred thousand phHSCs per well were plated in 6-well plates. After overnight serum starvation the cells were treated with either vehicle or $30 \mathrm{nM}$ of AZD3355 in DMEM with $0.1 \%$ BSA for 72 hours. Cells were harvested and total RNA was extracted using RNeasy Mini Kit as described in RT-quantitative PCR method. The purity of RNA (RNA integrity number or RIN) was assessed using 2100 Bioanalyzer instrument (Agilent, CA). To prepare high quality sequencing library from mRNA we were used TruSeq Stranded mRNA library prep kit (Illumina, CA) according to manufacture protocol. Sequencing was performed on NextSeq Hi (Illumnina, CA) sequencing instrument with setting at following output: R1=100; index=8; R2=100. RNASeq raw data were processed using FAST QC software (http://www.bioinformatics.babraham.ac.uk/projects/fastqc/) and aligned with STAR aligner ${ }^{62}$ via a computational pipeline implemented in NextFlow ${ }^{63}$. After alignment and summarization with featureCounts, data was normalized and differential expression was carried out with DESeq2 ${ }^{64}$. DEGs were considered significant at FDR $<0.1$. Annotation of enriched gene pathways was performed using ClusterProfiler ${ }^{65}$. Genes identified from the RNASeq were uploaded into the eXpression2Kinases (X2K) web tool ${ }^{24}$ a pipeline consisting of transcription factor enrichment analysis, protein-protein interaction network generation, and kinase enrichment analysis. Principle Components Analysis (PCA) was performed on counts after applying a variance-stabilizing transformation and summarized using pcaExplorer ${ }^{66}$.

\section{Human precision-cut liver slices:}

Human precision-cut liver slices (hPCLS) were generated from discarded remnants of surgically resected human livers ${ }^{67}$ that lacked patient identifiers, following Institutional Review Board (IRB) approval at Icahn School of Medicine at Mount Sinai. Three different patient backgrounds for liver samples were selected for the hPCLS study, of which 1 was male, 2 were female (Table 1). Normal-appearing resection margins surrounding HCC or metastatic regions were selected for hPCLS generation. The ischemic time between post-hepatectomy and generated PCLS was 3 - 4 hours. The resected liver pieces were transported to the lab in ice-cold Krebs-Henseleit buffer. Cores of $8 \mathrm{~mm}$ diameter were generated by using a stainlesssteel coring tool (Alabama Research and Development, AL), and the liver core was attached to the specimen holder by solvent- free cyanoacrylate adhesive (Best Klebstoffe GmbH \& Co. Germany). The liver core attached to the specimen holder was mounted in the buffer tray, which was submerged in carbogen saturated ice-cold Krebs-Henseleit buffer supplemented with $25 \mathrm{mM}$ glucose, and the liver slices were created using VT1200S tissue slicer (Leica Biosystem, IL). During slicing Krebs-Henseleit buffer was continuously supplied with carbogen. Intact liver slices were collected from the buffer tray using a soft bristle brush and transferred to six well tissue culture plate ( 3 slices/well) filled with $37^{\circ} \mathrm{C}$ warm William's E GlutaMAX (WE-GlutaMAX) media (Thermo Fisher Scientific, MA) supplemented with $25 \mathrm{mM}$ glucose and $50 \mu \mathrm{g} / \mathrm{ml}$ gentamycin (Thermo Fisher Scientific, MA). The slices were pre-incubated in a $37^{\circ} \mathrm{C}$ incubator supplemented with $5 \% \mathrm{CO}_{2}$ and $95 \% \mathrm{O}_{2}$ on a gentle rocker (10 rpm) for four hours to equilibrate the tissues. After four hours pre-incubation, fresh medium was added containing either vehicle (DMSO) or two different concentration of AZD3355 for additional 24 hours. At the end of drug treatment from each condition slices were used for total mRNA extraction. COL 1A1, aSMA and tumor necrosis factor 
alpha ( TNFa) were quantified using RT-quantitative PCR. For assessment of secreted Col1a1, culture media from each condition were collected, snap frozen and measured by ELISA as described previously ${ }^{61}$. For cytotoxicity assessment the luminescence signal of secreted lactate dehydrogenase (LDH) in media was measured using a Synergy HT (BioTek Instrument Inc., VT) luminometer using LDH-Glo cytotoxicity assay kit (Promega, WI) according to the instructions and purified lactate dehydrogenase from rabbit muscle as standard or positive control.

\section{In vivo assessment of AZD3355 efficacy in a murine NASH model:}

The animal protocol was approved by the Institutional Animal Care and Use Committee (IACUC) at the Icahn School of Medicine at Mount Sinai, NY (IACUC-2018-0060). Six week old male and female C57BL/6J mice (housed separately) were purchased from Jackson Laboratories (Farmington, CT). Five mice per cage were housed in a Helicobacter-free room for 12 hours light - 12 hours dark cycle and weighed once weekly.

Carbon tetrachloride $\left(\mathrm{CCl}_{4}\right)$ was purchased from Sigma-Aldrich, $\mathrm{MO} . \mathrm{CCl}_{4}$ was freshly dissolved in corn oil at final concentration of $5 \%$ before injection. The final dose of pure $\mathrm{CCl}_{4}$ was $0.2 \mu \mathrm{l} / \mathrm{g}$ of body weight of mice, delivered intraperitoneally once/week starting from initiation of the western diet/sugar water feeding and continued for a total period of 24 weeks. Western diet containing $21.2 \%$ fat (42\% Kcal), $41 \%$ sucrose and $1.25 \%$ cholesterol by weight was purchased from Envigo, WI (Teklad Custom diet). Sugar water solution contained $18.9 \mathrm{~g} / \mathrm{L} \mathrm{D-(+)-Glucose} \mathrm{(Sigma-Aldrich,} \mathrm{MO)} \mathrm{and} 23.1 \mathrm{~g} / \mathrm{L}$ D-(-)-Fructose (Sigma-Aldrich, MO) dissolved in autoclaved water and filter sterilized. The diet and sugar water were replaced twice weekly.

Methylcellulose $(4,000 \mathrm{cP}$ ) (Sigma-Aldrich, MO) diluted in UltraPure Distilled water (ThermoFisher Scientific) to $0.5 \% \mathrm{w} / \mathrm{v}$ and maintained at $4^{\circ} \mathrm{C}$, was used as a drug delivery vehicle. Two different concentrations of AZD3355 (2 mg/ml (w/v) dilution for $10 \mathrm{mg} / \mathrm{kg}$ and $6 \mathrm{mg} / \mathrm{ml}(\mathrm{w} / \mathrm{v})$ dilution for $30 \mathrm{mg} / \mathrm{kg}$ dosing) were made in $0.5 \%$ methylcellulose before each gavage. Obeticholic acid (OCA; FW 420.63) was used as a positive control, purchased from ApexBio (Houston, TX). OCA was also made fresh in $0.5 \%$ methylcellulose $(6 \mathrm{mg} / \mathrm{ml}(\mathrm{w} / \mathrm{v})$; OCA dilution for $30 \mathrm{mg} / \mathrm{kg}$ dosing) each week, aliquoted and stored at $-20^{\circ} \mathrm{C}$.

The experimental scheme of AZD3355 treatment in the FAT-NASH model is summarized in Suppl Figure S1. Both male and female C57BL/6J mice were fed a Western diet and sugar water, combined with weekly $\mathrm{CCl}_{4}$ injection via IP for 24 weeks. At week 12, five mice from each sex were sacrificed and NASH activity was assessed by: i) liver enzyme and lipid panel analysis in blood serum level, ii) pro-fibrotic gene and protein expression, iii) hepatic collagen quantification and iv) NAFLD activity scoring (data not shown). Beginning at week 13, the mice were randomly divided into five groups (9 males and 8 females per group) and treated for an additional 12 weeks as follows: i) $0.5 \%$ methylcellulose as vehicle, ii) AZD3355 at 10 $\mathrm{mg} / \mathrm{kg}$ body weight, iii) AZD3355 at $30 \mathrm{mg} / \mathrm{kg}$ body weight, iv) Obeticholic acid at $30 \mathrm{mg} / \mathrm{kg}$ body weight (as positive treatment of $\mathrm{NASH}$ ) and v) NASH background disease-control mice (5 mice) - only diet and $\mathrm{CCl}_{4}$ without vehicle or drug (group v data are not shown). The mice were dosed BID in 12-hour intervals for vehicle and AZD3355 (5 days/week) and QD for OCA (5 days/week) via gavage. Weekly body weight, WD and sugar water consumption were also recorded in each group. At the end of the experiment the animals from each group were sacrificed, with blood and liver collected for analyses.

At the termination of the study, blood samples were collected through the inferior vena cava for serum preparation. The liver and spleen were excised, cleaned in 1X PBS, and weights recorded. A small piece from the left liver lobe of each animal was excised and preserved in RNAlater (Qiagen, MD) for mRNA quantification. Another small piece from the left lobe was snapfrozen in liquid nitrogen for protein extraction. A third small piece from the left lobe was fixed in $10 \%$ formalin.

\section{Blood serum preparation, liver enzymes and lipid panel analysis:}

For blood serum preparation collected blood samples were kept at room temperature for 30 minutes to allow clotting. Serum was collected (top layer) after centrifugation at $2000 \mathrm{xg}$ for 10 minutes at $4^{\circ} \mathrm{C}$. Aspartate aminotransferase (AST), 
alanine aminotransferase (ALT) and triglycerides were measured from serum using an ARCHITECT c16000 Clinical Chemistry Analyzer (Abbott Diagnostics, MA) in the Mount Sinai Clinical Chemistry Laboratory facility according to the manufacturer's instruction.

\section{Picrosirius red staining for collagen quantification in liver sections:}

Liver was fixed in $10 \%$ formalin buffer and paraffin embedded liver tissues were sectioned using a $4 \mu \mathrm{m}$ microtome. Slides containing tissue sections were baked at $60^{\circ} \mathrm{C}$ for 1 hour and re-hydrated through xylene followed by graded ethanol (100\%, $95 \%, 85 \%$ and $70 \%$ ) into distilled water and processed for picrosirius red/Fast green staining. For collagen staining, rehydrated slides were stained for one hour in saturated picric acid with $0.1 \%$ Sirius Red (Direct Red-80; Sigma-Aldrich, MO) followed by counterstain with $0.01 \%$ Fast Green (Sigma-Aldrich, MO) for another hour. The slides were removed from the stain, rinsed in water and rapidly dehydrated through graded ethanol (70\%, 85\%, 95\% and 100\%) followed by xylene and finally placed on cover slips in Permount (ThermoFisher Scientific, NJ). Whole slides with staining sections were digitally scanned in an Aperio AT2 digital scanner (Leica Biosystems Inc., IL). The image from each scanned section was randomly saved as 5x zoom level (3 images/section) in Aperio ImageScope histopathological diagnostic software [v12.4.0.5043] (Leica Biosystems Inc., IL). A total of 6 sections/animal were stained and 3 images from each section (total 18 pictures/animal) were evaluated using BIOQUANT image analysis software (Bioquant Image Analysis Corporation, TN) to quantify collagen accumulation in liver tissue.

\section{H\&E staining and histopathological scoring of liver sections:}

A total of 2 sections/animal were stained with H\&E. Steatosis, hepatocyte ballooning, lobular inflammation, portal inflammation and fibrosis in liver sections were scored according to the NASH Clinical Research Network (NASH CRN) scoring system in a blinded fashion by an expert hepato-pathologist. NAFLD activity score (NAS), ranging from 0-8, was calculated ${ }^{28}$ based on the sum of scores of steatosis (0-3), hepatocyte ballooning (0-2) and lobular inflammation (0-3). Fibrosis (0-4) or portal inflammation (0-3) scores were assesed separately and not included in NAS.

\section{RT-quantitative PCR in liver tissue:}

Total mRNA was extracted using the RNeasy Mini Kit (Qiagen, CA). In brief, 20 mg of liver tissue was lysed in $400 \mu$ l RLTbuffer combined with $\beta$-mercaptoethanol. Lysates were homogenized in the presence of $5 \mathrm{~mm}$ stainless steel beads (Qiagen, Germantown, MD) using a TissueLyser LT homogenizer (Qiagen, Germantown, MD) at $50 \mathrm{~Hz} /$ second for 2 minutes. The tissue lysate was passed through QIAshredder column (Qiagen, CA) by spinning the column at high speed for 1 minute. The flow through was collected and mRNA was purified. RT-qPCR was performed as described above. Glyceraldehyde-3phosphate dehydrogenase (GAPDH) was used as a housekeeping gene to normalize expression.

\section{Protein quantification in liver tissue:}

Total protein was extracted from $20 \mathrm{mg}$ of liver tissue using RIPA buffer (50 mM Tris- $\mathrm{HCl}$ pH8.0, $150 \mathrm{mM} \mathrm{NaCl}, 1 \%$ IGEPAL, 0.5 \% Sodium Deoxycholate and $0.1 \%$ SDS) along with Pierce Protease Inhibitor Mini Tablets, EDTA-Free (Thermo Scientific, IL) and phosphatase inhibitor Cocktail (Thermo Scientific, IL). Total protein was collected from the homogenate (middle aqueous phase) after centrifugation at $14,000 \mathrm{rpm}$ for 10 minutes at $4^{\circ} \mathrm{C}$, and measured by Bradford colorimetric assay using Protein Assay Dye Reagent Concentrate (Bio-Rad, CA). $15 \mu \mathrm{g}$ of proteins were loaded in NuPAGE 4-12 \% Bis-Tris gels (Thermo Scientific, IL). After transfer to the PVDF membrane the blot was blocked with $5 \%$ non-fat milk in 1X PBS. The primary antibodies were rabbit anti-Collagen1 (Bioss, MA), rabbit anti-aSMA (Abcam, MA) and mouse anti-GAPDH (Millipore, CA). After hybridization with HRP-conjugated secondary antibody (either Goat anti-rabbit HRP (Jackson ImmunoResearch Laboratories, PA) or anti-mouse IgG-HRP (Cell Signaling Technology, MA) the membrane was treated with Immobilon Western Chemiluminescent HRP substrate (Millipore, MA) and the signals were captured with Amersham Imager 6000 (GE Healthcare, PA). Protein bands of $210 \mathrm{kD}$ for Collagen $1 \mathrm{a} 1$ and $42 \mathrm{kD}$ for aSMA were recognized by the respective antibodies. A $37 \mathrm{kD}$ band of GAPDH was quantified as the loading control. For densitometric measurement of the protein 
bands, images were exported and analyzed using ImageJ 1.50f software (http://imagej.nih.gov/ij) and bands were normalized to the loading control GAPDH.

\section{Statistical analysis:}

Data analysis was performed using GraphPad Prism v8.0.1 statistical software (GraphPad Software, Inc., CA). Standard error mean $( \pm S E M)$ was calculated according to unpaired two tailed Mann-Whitney test where Gaussian distribution is nonparametric. Unless otherwise specified, $\mathrm{p}$ values $<0.05$ were considered statistically significant vs vehicle group.

\section{Institutional Approvals:}

All animal studies were approved by the Mount Sinai IACUC. Use of human tissues for precision cut-liver slices was IRBexempt in accordance with guidelines of the Mount Sinai Institutional Review Board, as there were no patient-identifiers and the tissue was otherwise intended to be discarded.

All other methods were performed in accordance with the relevant institutional guidelines and regulations. The study is the study is reported in accordance with ARRIVE guidelines ${ }^{68}$.

\section{Declarations}

\section{Acknowledgements}

This study was supported by grant from the NIH (NCATS 4UH3TR002077). The original connectivity mapping studies and the PCLS experiments were supported by funds from Astra-Zeneca. We thank Dr. Shuang Wang and James Carter for technical assistance.

\section{Author contributions:}

Dipankar Bhattacharya: Study design, liver cell, human liver slice culture and mice studies,

manuscript and figure preparation

Christine Becker: Data generation for drug repurposing, data analysis, manuscript preparation

Benjamin Readhead: Study design, data generation for drug repurposing analysis, data analysis, manuscript preparation

Nicolas Goossens: Data generation for drug repurposing analysis, data analysis, manuscript preparation

Jacqueline Novik: Data generation for drug repurposing analysis, data analysis

Maria Isabel Fiel: Pathologic assessment of liver, manuscript preparation

Leslie P. Cousens: Study design, data generation for drug repurposing analysis, data analysis, manuscript preparation

Björn Magnusson: Data generation for drug repurposing analysis, data analysis

Anna Backmark: Data generation for drug repurposing analysis, data analysis

Ryan Hicks: Data generation for drug repurposing analysis, data analysis

Joel T. Dudley: Overall study design and interpretation, data analysis, manuscript preparation

Scott L. Friedman: Overall study design and interpretation, data analysis, manuscript preparation and final review, submission 


\section{Conflict of interest:}

Leslie P. Cousens, Björn Magnusson, Anna Backmark and Ryan Hicks are employees of AstraZeneca Pharmaceuticals

Financial Support: This study was supported by NIH, NCATS Grant number 4UH3TR002077 to Joel T. Dudley and Scott L. Friedman

\section{References}

1 Paik, J. M. et al. Mortality Related to Nonalcoholic Fatty Liver Disease Is Increasing in the United States. Hepatol Commun3, 1459-1471, doi:10.1002/hep4.1419 (2019).

2 Younossi, Z. M. et al. Burden of Illness and Economic Model for Patients With Nonalcoholic Steatohepatitis in the United States. Hepatology69, 564-572, doi:10.1002/hep.30254 (2019).

3 Younossi, Z. et al. Global Perspectives on Nonalcoholic Fatty Liver Disease and Nonalcoholic Steatohepatitis. Hepatology69, 2672-2682, doi:10.1002/hep.30251 (2019).

4 Friedman, S. L., Neuschwander-Tetri, B. A., Rinella, M. \& Sanyal, A. J. Mechanisms of NAFLD development and therapeutic strategies. Nat Med24, 908-922, doi:10.1038/s41591-018-0104-9 (2018).

5 Kanwal, F. et al. Risk of Hepatocellular Cancer in Patients With Non-Alcoholic Fatty Liver Disease. Gastroenterology155, 1828-1837 e1822, doi:10.1053/j.gastro.2018.08.024 (2018).

6 Cholankeril, G. et al. Temporal Trends Associated With the Rise in Alcoholic Liver Disease-related Liver Transplantation in the United States. Transplantation103, 131-139, doi:10.1097/TP.0000000000002471 (2019).

7 Younossi, Z. M. et al. Obeticholic acid for the treatment of non-alcoholic steatohepatitis: interim analysis from a multicentre, randomised, placebo-controlled phase 3 trial. Lancet394, 2184-2196, doi:10.1016/S0140-6736(19)33041-7 (2019).

8 Dowden, H. \& Munro, J. Trends in clinical success rates and therapeutic focus. Nat Rev Drug Discov18, 495-496, doi:10.1038/d41573-019-00074-z (2019).

9 Morgan, P. et al. Impact of a five-dimensional framework on R\&D productivity at AstraZeneca. Nat Rev Drug Discov17, 167181, doi:10.1038/nrd.2017.244 (2018).

10 Hwang, T. J. et al. Failure of Investigational Drugs in Late-Stage Clinical Development and Publication of Trial Results. JAMA internal medicine176, 1826-1833, doi:10.1001/jamainternmed.2016.6008 (2016).

11 Fogel, D. B. Factors associated with clinical trials that fail and opportunities for improving the likelihood of success: A review. Contemp Clin Trials Commun11, 156-164, doi:10.1016/j.conctc.2018.08.001 (2018).

12 Peon, A., Naulaerts, S. \& Ballester, P. J. Predicting the Reliability of Drug-target Interaction Predictions with Maximum Coverage of Target Space. Sci Rep7, 3820, doi:10.1038/s41598-017-04264-w (2017).

13 Jahchan, N. S. et al. A drug repositioning approach identifies tricyclic antidepressants as inhibitors of small cell lung cancer and other neuroendocrine tumors. Cancer discovery3, 1364-1377, doi:10.1158/2159-8290.CD-13-0183 (2013).

14 Dudley, J. T. et al. Computational repositioning of the anticonvulsant topiramate for inflammatory bowel disease. Sci Trans/ Med3, 96ra76, doi:10.1126/scitransImed.3002648 (2011). 
15 Talevi, A. \& Bellera, C. L. Challenges and opportunities with drug repurposing: finding strategies to find alternative uses of therapeutics. Expert opinion on drug discovery15, 397-401, doi:10.1080/17460441.2020.1704729 (2020).

16 Wooden, B., Goossens, N., Hoshida, Y. \& Friedman, S. L. Using Big Data to Discover Diagnostics and Therapeutics for Gastrointestinal and Liver Diseases. Gastroenterology152, 53-67 e53, doi:10.1053/j.gastro.2016.09.065 (2017).

17 Bredenoord, A. J. Lesogaberan, a GABA(B) agonist for the potential treatment of gastroesophageal reflux disease. IDrugs12, 576-584 (2009).

18 Boeckxstaens, G. E. et al. Effects of lesogaberan on reflux and lower esophageal sphincter function in patients with gastroesophageal reflux disease. Gastroenterology139, 409-417, doi:10.1053/j.gastro.2010.04.051 (2010).

19 Miner, P. B., Jr., Silberg, D. G., Ruth, M., Miller, F. \& Pandolfino, J. Dose-dependent effects of lesogaberan on reflux measures in patients with refractory gastroesophageal reflux disease: a randomized, placebo-controlled study. BMC Gastroentero/14, 188, doi:10.1186/1471-230X-14-188 (2014).

20 Sirota, M. et al. Discovery and preclinical validation of drug indications using compendia of public gene expression data. Sci Trans/ Med3, 96ra77, doi:10.1126/scitranslmed.3001318 (2011).

21 Wang, Z. et al. Extraction and analysis of signatures from the Gene Expression Omnibus by the crowd. Nature communications7, 12846, doi:10.1038/ncomms12846 (2016).

$22 \mathrm{Xu}$, L. et al. Human hepatic stellate cell lines, LX-1 and LX-2: new tools for analysis of hepatic fibrosis. Gut54, 142-151, doi:10.1136/gut.2004.042127 (2005).

23 Blaner, W. S. et al. Hepatic stellate cell lipid droplets: A specialized lipid droplet for retinoid storage. Biochim Biophys Acta1791, 467-473, doi:S1388-1981(08)00207-2 [pii]

10.1016/j.bbalip.2008.11.001 (2009).

24 Clarke, D. J. B. et al. eXpression2Kinases (X2K) Web: linking expression signatures to upstream cell signaling networks. Nucleic Acids Res46, W171-W179, doi:10.1093/nar/gky458 (2018).

25 Paish, H. L. et al. A Bioreactor Technology for Modeling Fibrosis in Human and Rodent Precision-Cut Liver Slices. Hepatology, doi:10.1002/hep.30651 (2019).

26 Tsuchida, T. et al. A Simple Diet- and Chemical-Induced Murine NASH Model with Rapid Progression of Steatohepatitis, Fibrosis and Liver Cancer. J Hepatol, doi:10.1016/j.jhep.2018.03.011 (2018).

27 Carter, J. K. et al. Modeling dysbiosis of human NASH in mice: Loss of gut microbiome diversity and overgrowth of Erysipelotrichales. PLoS One16, e0244763, doi:10.1371/journal.pone.0244763 (2021).

28 Kleiner, D. E. et al. Design and validation of a histological scoring system for nonalcoholic fatty liver disease. Hepatology41, 1313-1321, doi:10.1002/hep.20701 (2005).

29 Lehmann, A. GABAB receptors as drug targets to treat gastroesophageal reflux disease. Pharmacol Ther122, 239-245, doi:10.1016/j.pharmthera.2009.02.008 (2009).

30 Branden, L., Fredriksson, A., Harring, E., Jensen, J. \& Lehmann, A. The novel, peripherally restricted GABAB receptor agonist lesogaberan (AZD3355) inhibits acid reflux and reduces esophageal acid exposure as measured with 24-h pHmetry in dogs. Eur J Pharmaco/634, 138-141, doi:10.1016/j.ejphar.2010.02.015 (2010). 
31 Boeckxstaens, G. E. et al. A novel reflux inhibitor lesogaberan (AZD3355) as add-on treatment in patients with GORD with persistent reflux symptoms despite proton pump inhibitor therapy: a randomised placebo-controlled trial. Gut60, 1182-1188, doi:10.1136/gut.2010.235630 (2011).

32 Friedman, S. L., Roll, F. J., Boyles, J. \& Bissell, D. M. Hepatic lipocytes: the principal collagen-producing cells of normal rat liver. Proc Natl Acad Sci U S A82, 8681-8685, doi:10.1073/pnas.82.24.8681 (1985).

33 Tsuchida, T. \& Friedman, S. L. Mechanisms of hepatic stellate cell activation. Nat Rev Gastroenterol Hepato/14, 397-411, doi:10.1038/nrgastro.2017.38 (2017).

34 Xiao, F. et al. The GABAB receptor inhibits activation of hepatic stellate cells. Dig Dis Sci55, 261-267, doi:10.1007/s10620-009-0743-2 (2010).

35 Potter, J. J., Rennie-Tankersley, L., Anania, F. A. \& Mezey, E. A transient increase in c-myc precedes the transdifferentiation of hepatic stellate cells to myofibroblast-like cells. Liver19, 135-144 (1999).

36 Cai, X. et al. CXCL6-EGFR-induced Kupffer cells secrete TGF-beta1 promoting hepatic stellate cell activation via the SMAD2/BRD4/C-MYC/EZH2 pathway in liver fibrosis. J Cell Mol Med22, 5050-5061, doi:10.1111/jcmm.13787 (2018).

37 Chen, H., Liu, H. \& Qing, G. Targeting oncogenic Myc as a strategy for cancer treatment. Signal Transduct Target Ther3, 5, doi:10.1038/s41392-018-0008-7 (2018).

38 Zhao, S. F., Wang, S. G., Zhao, Z. Y. \& Li, W. L. AKR1C1-3, notably AKR1C3, are distinct biomarkers for liver cancer diagnosis and prognosis: Database mining in malignancies. Oncol Lett18, 4515-4522, doi:10.3892/ol.2019.10802 (2019).

39 Hsieh, S. Y. et al. Identifying apoptosis-evasion proteins/pathways in human hepatoma cells via induction of cellular hormesis by UV irradiation. J Proteome Res8, 3977-3986, doi:10.1021/pr900289g (2009).

40 Guo, P. et al. Ribosomal protein S15a promotes tumor angiogenesis via enhancing Wnt/beta-catenin-induced FGF18 expression in hepatocellular carcinoma. Oncogene37, 1220-1236, doi:10.1038/s41388-017-0017-y (2018).

41 Gunasekaran, V. P. \& Ganeshan, M. Inverse correlation of ribosomal protein S27A and multifunctional protein YB-1 in hepatocellular carcinoma. Clin Biochem47, 1262-1264, doi:10.1016/j.clinbiochem.2014.05.004 (2014).

42 Zhang, D. Y. et al. A hepatic stellate cell gene expression signature associated with outcomes in hepatitis C cirrhosis and hepatocellular carcinoma after curative resection. Gut65, 1754 - 1764, doi:10.1136/gutjnl-2015-309655 (2016).

43 Hoshida, Y. et al. Gene expression in fixed tissues and outcome in hepatocellular carcinoma. N Engl J Med359, 19952004, doi:NEJMoa0804525 [pii]

10.1056/NEJMoa0804525 (2008).

44 Verbeke, L. et al. FXR agonist obeticholic acid reduces hepatic inflammation and fibrosis in a rat model of toxic cirrhosis. Sci Rep6, 33453, doi:10.1038/srep33453 (2016).

45 Adorini, L., Pruzanski, M. \& Shapiro, D. Farnesoid X receptor targeting to treat nonalcoholic steatohepatitis. Drug Discov Today17, 988-997, doi:10.1016/j.drudis.2012.05.012 (2012).

46 Harrison, S. A. et al. Selonsertib for patients with bridging fibrosis or compensated cirrhosis due to NASH: Results from randomized phase III STELLAR trials. J Hepato/73, 26-39, doi:10.1016/j.jhep.2020.02.027 (2020).

47 Ratziu, V. \& Friedman, S. L. Why do so many NASH trials fail? Gastroenterology, doi:10.1053/j.gastro.2020.05.046 (2020). 
48 Natri, H. M., Wilson, M. A. \& Buetow, K. H. Distinct molecular etiologies of male and female hepatocellular carcinoma. BMC Cancer19, 951, doi:10.1186/s12885-019-6167-2 (2019).

49 Lonardo, A. et al. Sex Differences in Nonalcoholic Fatty Liver Disease: State of the Art and Identification of Research Gaps. Hepatology70, 1457-1469, doi:10.1002/hep.30626 (2019).

50 Vandel, J. et al. Hepatic molecular signatures highlight the sexual dimorphism of Non-Alcoholic SteatoHepatitis (NASH). Hepatology, doi:10.1002/hep.31312 (2020).

51 Balakrishnan, M. et al. Women have Lower Risk of Nonalcoholic Fatty Liver Disease but Higher Risk of Progression vs Men: A Systematic Review and Meta-analysis. Clin Gastroenterol Hepatol, doi:10.1016/j.cgh.2020.04.067 (2020).

52 Hicks, D. F. et al. Transcriptome-based repurposing of apigenin as a potential anti-fibrotic agent targeting hepatic stellate cells. Sci Rep7, 42563, doi:10.1038/srep42563 (2017).

53 Soule, H. D., Vazguez, J., Long, A., Albert, S. \& Brennan, M. A human cell line from a pleural effusion derived from a breast carcinoma. J Natl Cancer Inst51, 1409-1416, doi:10.1093/jnci/51.5.1409 (1973).

54 Subramanian, A. et al. A Next Generation Connectivity Map: L1000 Platform and the First 1,000,000 Profiles. Cel/171, 1437-1452 e1417, doi:10.1016/j.cell.2017.10.049 (2017).

55 Liu, W., Baker, S. S., Baker, R. D., Nowak, N. J. \& Zhu, L. Upregulation of hemoglobin expression by oxidative stress in hepatocytes and its implication in nonalcoholic steatohepatitis. PloS one6, e24363 (2011).

56 Feng, T. et al. Smad7 regulates compensatory hepatocyte proliferation in damaged mouse liver and positively relates to better clinical outcome in human hepatocellular carcinoma. Clin Sci (Lond)128, 761-774, doi:10.1042/CS20140606 (2015).

57 Subramanian, A. et al. Gene set enrichment analysis: a knowledge-based approach for interpreting genome-wide expression profiles. Proc Natl Acad Sci U S A102, 15545-15550, doi:10.1073/pnas.0506580102 (2005).

58 Alstermark, C. et al. Synthesis and pharmacological evaluation of novel gamma-aminobutyric acid type B (GABAB) receptor agonists as gastroesophageal reflux inhibitors. J Med Chem51, 4315-4320, doi:10.1021/jm701425k (2008).

59 Hong, F., Chou, H., Fiel, M. I. \& Friedman, S. L. Antifibrotic activity of sorafenib in experimental hepatic fibrosis: refinement of inhibitory targets, dosing, and window of efficacy in vivo. Dig Dis Sci58, 257-264, doi:10.1007/s10620-012-2325-y (2013).

60 Friedman, S. L. et al. Isolated hepatic lipocytes and Kupffer cells from normal human liver: morphological and functional characteristics in primary culture. Hepatology15, 234-243 (1992).

61 Bhattacharya, D. et al. Aramchol downregulates stearoyl CoA-desaturase 1 in hepatic stellate cells to attenuate cellular fibrogenesis. J HEP REPORTS, in press (2021).

62 Dobin, A. et al. STAR: ultrafast universal RNA-seq aligner. Bioinformatics29, 15-21, doi:10.1093/bioinformatics/bts635 (2013).

63 Di Tommaso, P. et al. Nextflow enables reproducible computational workflows. Nat Biotechno/35, 316-319, doi:10.1038/nbt.3820 (2017).

64 Love, M. I., Huber, W. \& Anders, S. Moderated estimation of fold change and dispersion for RNA-seq data with DESeq2. Genome Bio/15, 550, doi:10.1186/s13059-014-0550-8 (2014).

65 Yu, G., Wang, L. G., Han, Y. \& He, Q. Y. clusterProfiler: an R package for comparing biological themes among gene clusters. OMICS16, 284-287, doi:10.1089/omi.2011.0118 (2012). 
66 Marini, F. \& Binder, H. pcaExplorer: an R/Bioconductor package for interacting with RNA-seq principal components. BMC Bioinformatics20, 331, doi:10.1186/s12859-019-2879-1 (2019).

67 van de Bovenkamp, M. et al. Precision-cut liver slices as a new model to study toxicity-induced hepatic stellate cell activation in a physiologic milieu. Toxicol Sci85, 632-638, doi:10.1093/toxsci/kfi127 (2005).

68 Percie du Sert, N. et al. Reporting animal research: Explanation and elaboration for the ARRIVE guidelines 2.0. PLoS Bio/18, e3000411, doi:10.1371/journal.pbio.3000411 (2020).

\section{Tables}

Table 1. Characteristics of human liver donors for PCLS studies

\begin{tabular}{|llll|}
\hline Patient \# & Sex & Age (Years) & Diagnosis \\
\hline 1 & Female & 63 & Hilar cholangiocarcinoma (Non-viral) \\
\hline 2 & Female & 74 & HCV infected colorectal liver metastasis (NASH - Grade 1 Stage 2) \\
\hline 3 & Male & 60 & Fibrolamellar HCC (Non-viral) \\
\hline
\end{tabular}

Table 2. NAFLD activity score (NAS) and fibrosis stage of AZD3355 treated mice

\begin{tabular}{|c|c|c|c|c|c|c|c|c|}
\hline & Male & & & & Female & & & \\
\hline Treatment & Vehicle & $\begin{array}{l}\text { AZD3355 } \\
(10 \mathrm{mg} / \mathrm{kg})\end{array}$ & $\begin{array}{l}\text { AZD3355 } \\
(30 \mathrm{mg} / \mathrm{kg})\end{array}$ & $\begin{array}{l}\text { OCA } \\
(30 \mathrm{mg} / \mathrm{kg})\end{array}$ & Vehicle & $\begin{array}{l}\text { AZD3355 } \\
(10 \mathrm{mg} / \mathrm{kg})\end{array}$ & $\begin{array}{l}\text { AZD3355 } \\
(30 \mathrm{mg} / \mathrm{kg})\end{array}$ & $\begin{array}{l}\text { OCA } \\
(30 \mathrm{mg} / \mathrm{kg})\end{array}$ \\
\hline Steatosis & $\begin{array}{l}2.8 \pm \\
0.1\end{array}$ & $2.6 \pm 0.2$ & $2.3 \pm 0.2$ & $2.0 \pm 0.2$ & $\begin{array}{l}2.5 \pm \\
0.1\end{array}$ & $2.5 \pm 0.1$ & $2.2 \pm 0.1$ & $2.0 \pm 0.2$ \\
\hline $\begin{array}{l}\text { Hepatocyte } \\
\text { Ballooning }\end{array}$ & $\begin{array}{l}1.7 \pm \\
0.1\end{array}$ & $1.6 \pm 0.1$ & $1.7 \pm 0.1$ & $1.0 \pm 0.2$ & $\begin{array}{l}1.2 \pm \\
0.1\end{array}$ & $1.2 \pm 0.1$ & $1.2 \pm 0.1$ & $1.1 \pm 0.2$ \\
\hline $\begin{array}{l}\text { Lobular } \\
\text { Inflammation }\end{array}$ & $\begin{array}{l}2.7 \pm \\
0.1\end{array}$ & $2.7 \pm 0.1$ & $2.5 \pm 0.2$ & $1.7 \pm 0.2$ & $\begin{array}{l}2.3 \pm \\
0.1\end{array}$ & $2.5 \pm 0.1$ & $2.7 \pm 0.1$ & $1.6 \pm 0.1$ \\
\hline NAS & $\begin{array}{l}7.4 \pm \\
0.2\end{array}$ & $7.1 \pm 0.4$ & $6.6 \pm 0.6$ & $4.7 \pm 0.7$ & $\begin{array}{l}6.1 \pm \\
0.4\end{array}$ & $6.4 \pm 0.4$ & $6.4 \pm 0.3$ & $4.7 \pm 0.6$ \\
\hline $\begin{array}{l}\text { Fibrosis } \\
\text { stage }\end{array}$ & $\begin{array}{l}3.4 \pm \\
0.1\end{array}$ & $3.1 \pm 0.1$ & $3.0 \pm 0.0$ & $2.7 \pm 0.2$ & $\begin{array}{l}3.6 \pm \\
0.1\end{array}$ & $3.0 \pm 0.1$ & $3.1 \pm 0.1$ & $2.2 \pm 0.1$ \\
\hline
\end{tabular}

\section{Figures}




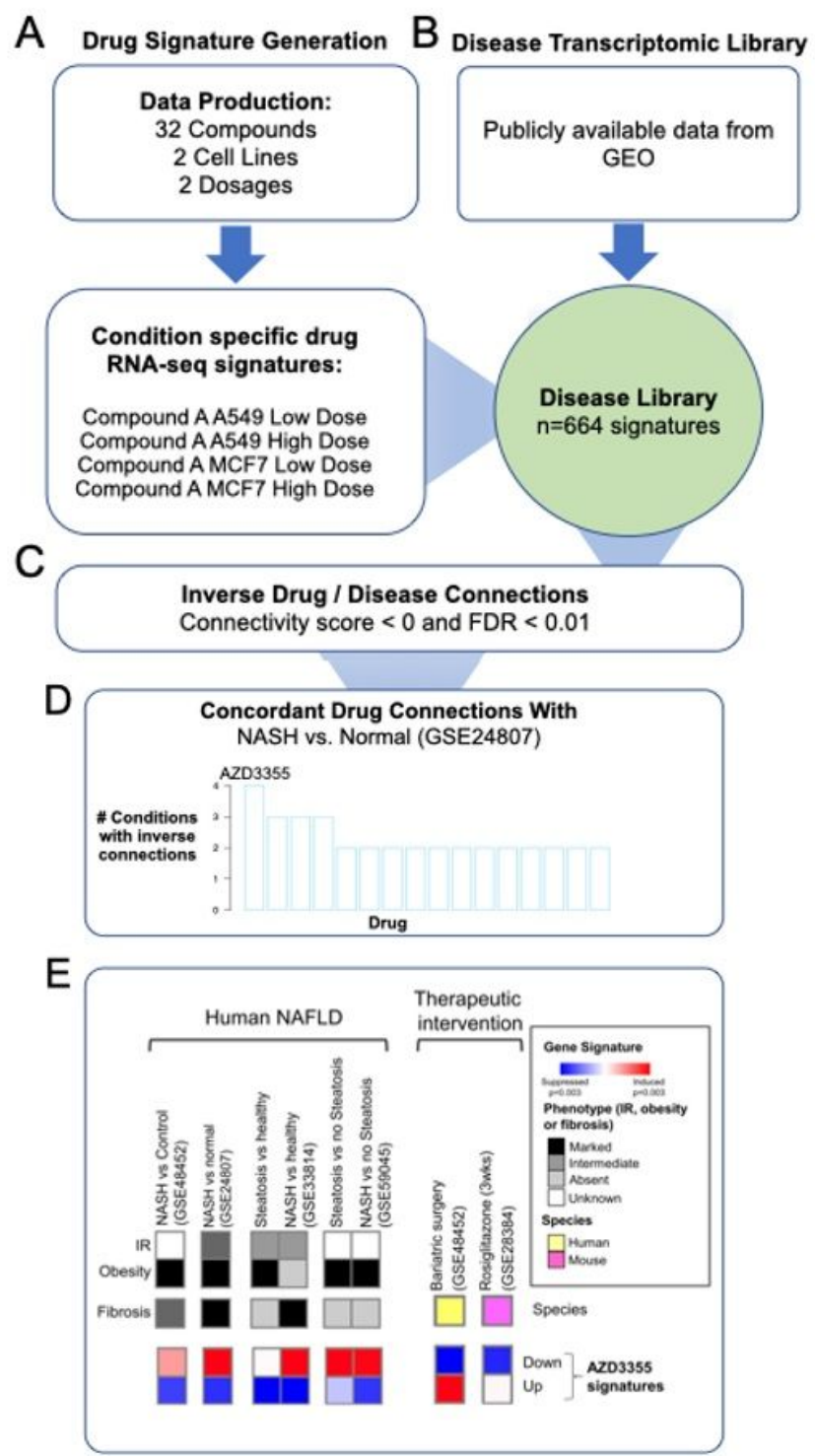

\section{Figure 1}

Computational drug repurposing identifying AZD3355 as a potential treatment for NASH. (A) We generated RNA-sequence profiles for 32 drugs, across four conditions to define condition specific drug signatures, (B) which were then compared with a large library of publicly available disease gene expression profiles using a connectivity mapping approach. (C-D) Significant (FDR < 0.01), inverse connections were found between all four AZD3355 signatures and a NASH gene expression signature (GSE24807). (E) In silico validation of AZD3355 in additional human and therapeutic NAFLD hepatic transcriptomic datasets. AZD3355 perturbation signatures were inversely enriched in human NAFLD (left), and positively enriched in datasets testing previously validated NAFLD therapies in human and mice (right). Abbreviations: IR, insulin resistance 
A
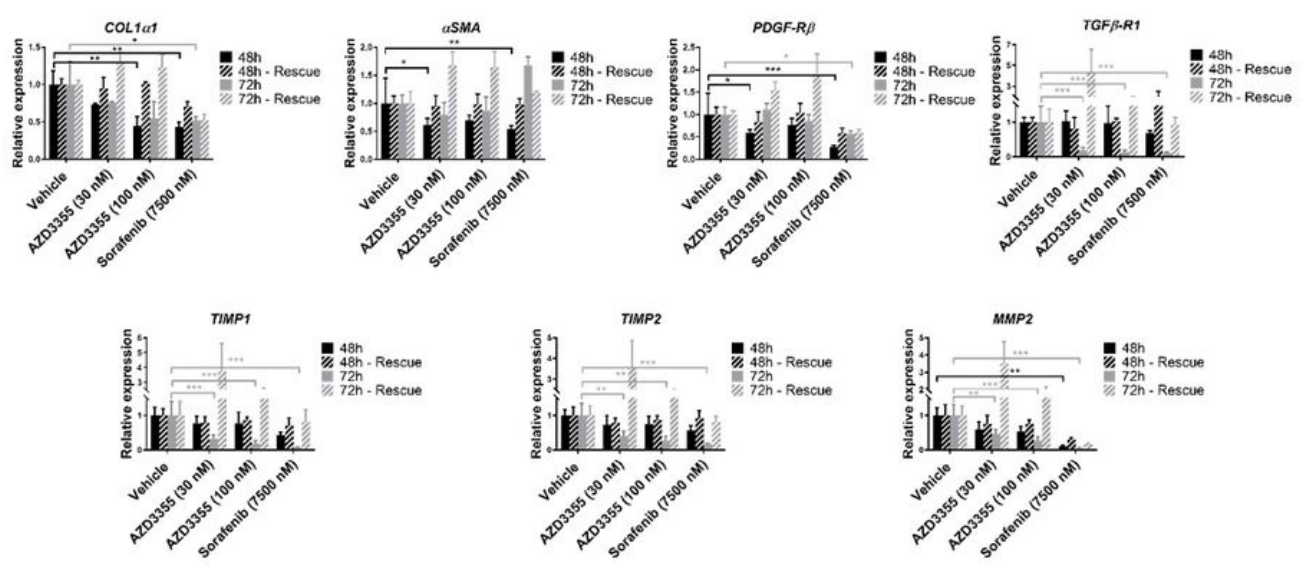

B
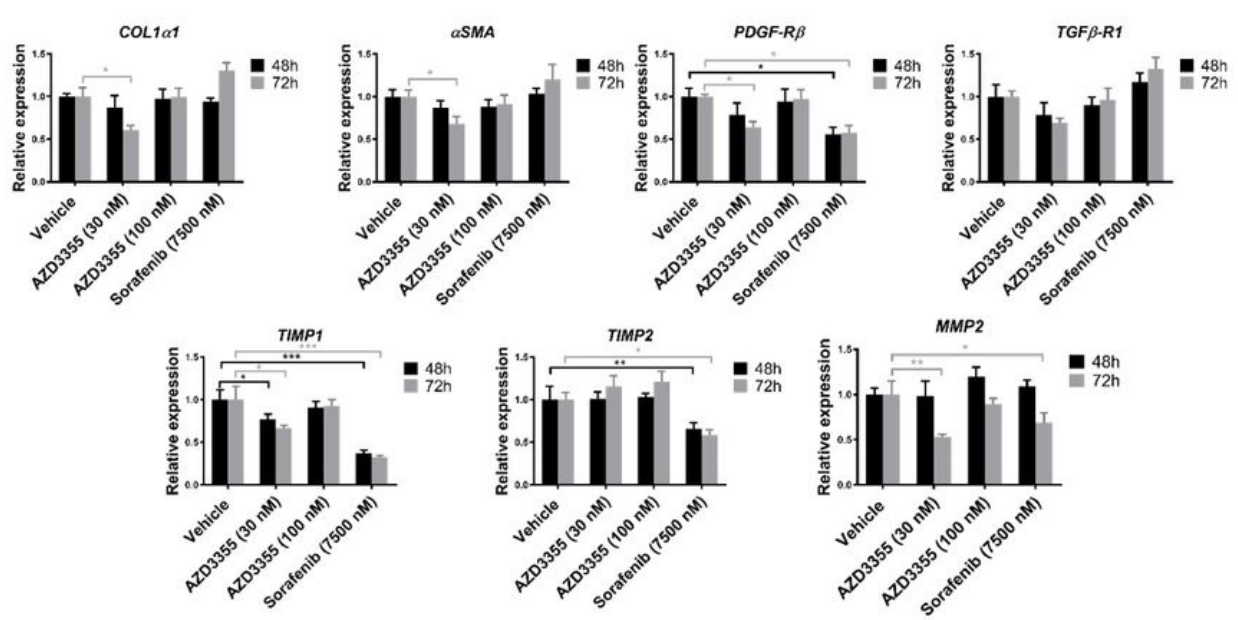

Figure 2

AZD3355 down-regulates activation-related mRNAs in HSCs. LX-2 cells (A) were treated with either DMSO (as vehicle) or either AZD3355 (30 nM or $100 \mathrm{nM}$ ) and 7,500 nM Sorafenib (as positive control) for 48 and 72 hours. The vehicle or drugs were withdrawn after 48 and 72 hours and cells were maintained in DMEM media with $10 \%$ FBS for an additional 48 and 72 hours, respectively. Dose-dependent down-regulation of fibrogenic related mRNA expression was observed in response to AZD3355 treatment. Recovery of gene expression in (striped bars) after drug removal (solid bars) indicates that downregulation was reversible and not due to toxicity. Primary human hepatic stellate cells (B) were treated as described for as LX-2 cells (without rescue). Stellate cell activated genes were significantly down-regulated in 30 nM AZD3355 at 72 hours treatment. Results are reported as means $\triangle$ SEM $(n=3) .{ }^{*} p<0.05,{ }^{* *} p<0.01,{ }^{* *} p<0.001$. 
A.

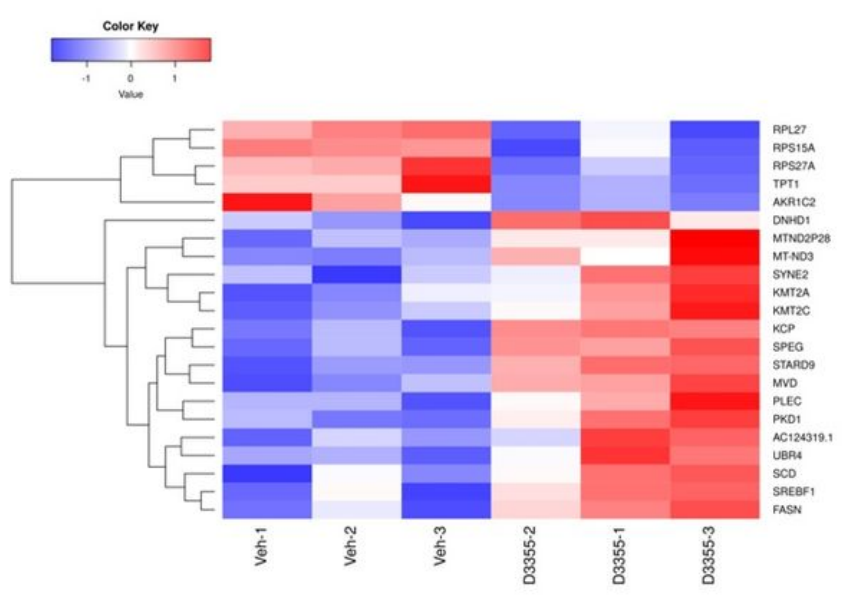

B.

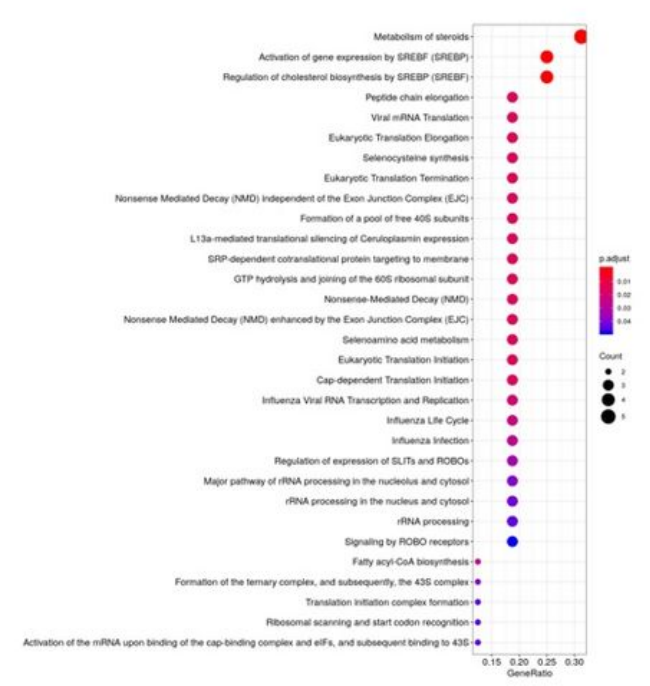

Figure 3

Differential gene expression and molecular pathway analysis in primary human hepatic stellate cells treated with AZD3355 Primary human hepatic stellate cells were treated with either DMSO (as vehicle) or AZD3355 (30 nM) for 72 hours. A heatmap was generated from RNA-seq data $(A)$ summarizing -significantly differentially expressed genes (padj $<0.1)$ in AZD3355 treated cells compared to vehicle control. Enrichment analysis of differentially expressed genes (B) reveals the top pathway identified: 'Metabolism of steroids', which contains the genes AKR1C2, SCD, MVD, SREBF1 and FASN. 
A

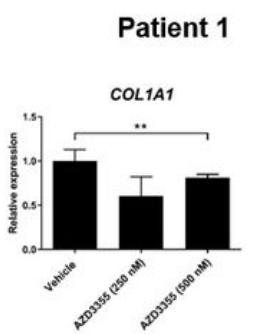

Patient 2

Patient 3
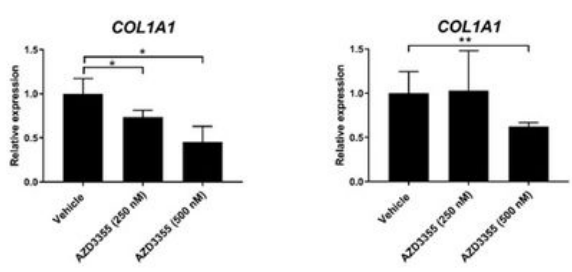

aSMA

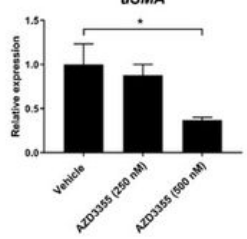

aSMA

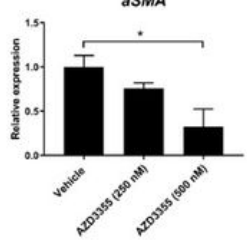

aSMA
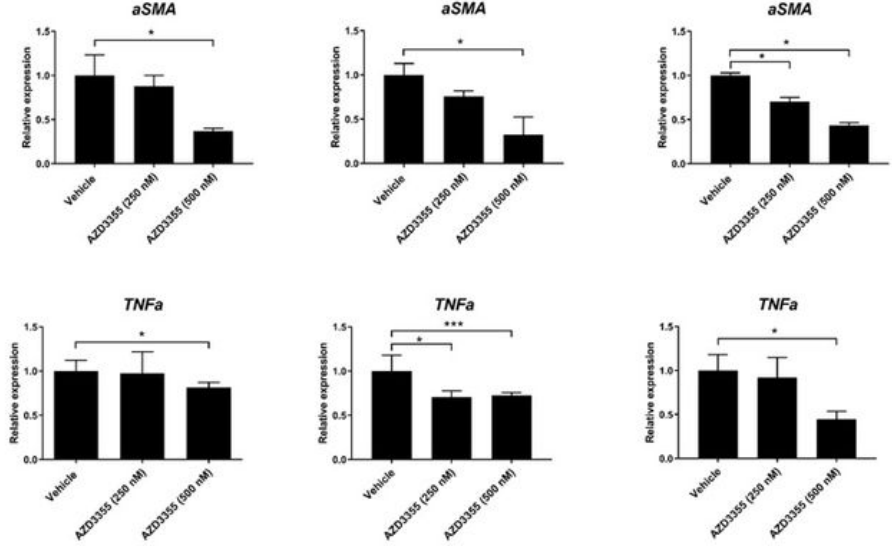

B

Patient 1

Patient 2

Patient 3
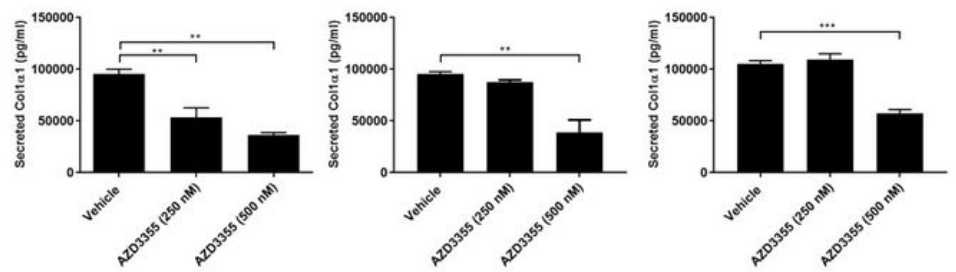

\section{Figure 4}

AZD3355 reduces fibrogenic and inflammatory gene expression as well as Col1冈1 protein secretion in human liver slices. Precision cut human liver slices (hPCLS) from three different patient donor liver samples were cultured with either DMSO (as vehicle) or AZD3355 (250 and $500 \mathrm{nM}$ ) for 24 hours. Quantitative PCR was used to measure mRNA expression for Col1A1, aSMA and TNFa. Two liver slices ( $8 \mathrm{~mm}$ diameter each) were used for each patient/drug concentration. Col1A1, aSMA and TNFa gene expression was significantly down-regulated by AZD3355 treatment in all three patient samples (A). Secreted Col1ه1 was measured from culture media by ELISA at the time of harvesting liver slices. Secreted Col1 $₫ 1$ protein in culture media was significantly reduced compared to vehicle group by AZD3355 treatment in all three patient liver slices (B).

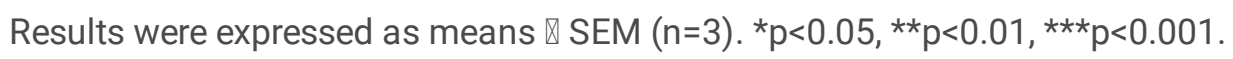


A
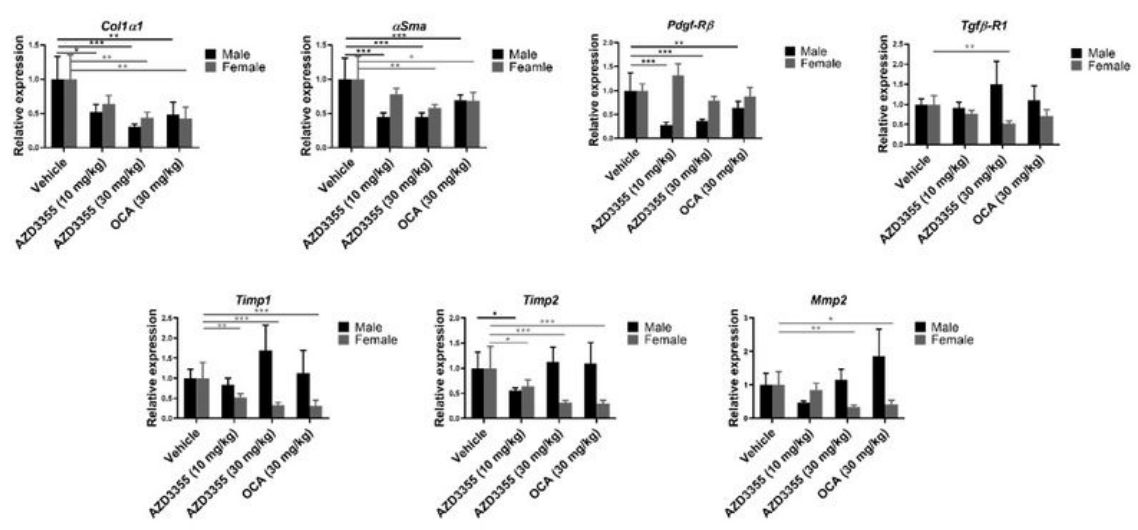

B

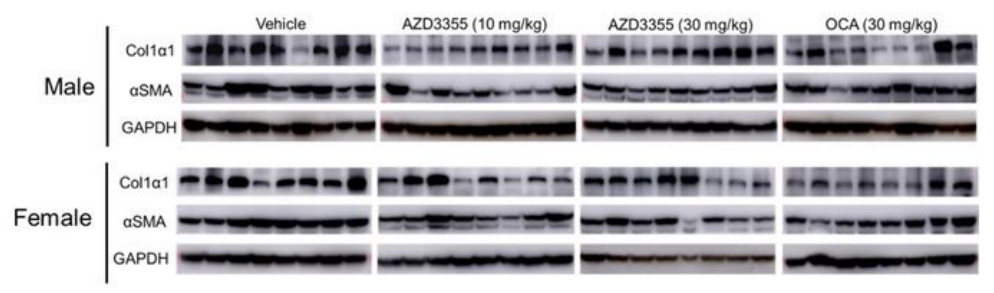

C
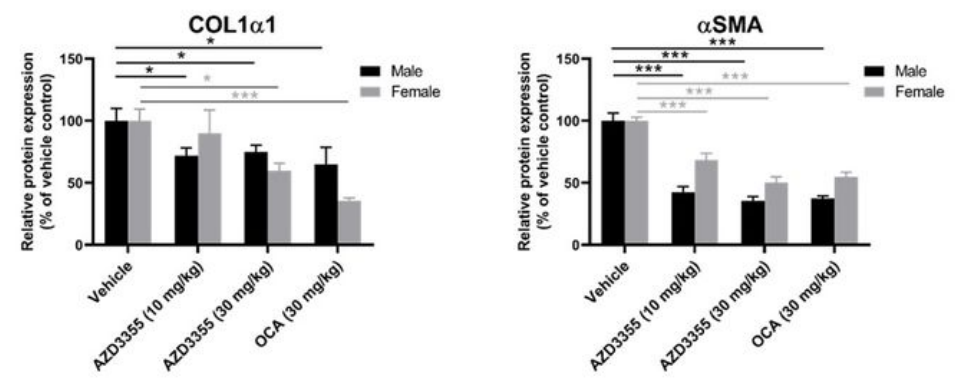

Figure 5

Expression of hepatic fibrogenic genes and proteins are down-regulated by AZD3355 in a murine NASH model. (A) Expression of fibrogenic mRNAs in male (black bar) or female (gray bar) murine liver tissues was quantified by RT-qPCR. Data was normalized to GAPDH and expressed relative to livers from the vehicle-treated group. (B) Western blots for Col1a1, aSMA and GAPDH in male and female mice from whole liver lysates. (C) Relative protein expression compared to the vehicle-treated group was quantified by densitometry of respective Western blots bands, and GAPDH was used as internal control. Vehicle: $\mathrm{n}=9$ male, 8 female; AZD3355 (10 mg/kg): $\mathrm{n}=9$ male, 8 female; AZD3355 (30 mg/kg): $\mathrm{n}=9$ male, 8 female; OCA (30 mg/kg): $\mathrm{n}=9$ male, 8 female animals. Results were expressed as means $\otimes S E M .{ }^{*} p<0.05,{ }^{* *} p<0.01$, $\star \star \star p<0.001$. 
A

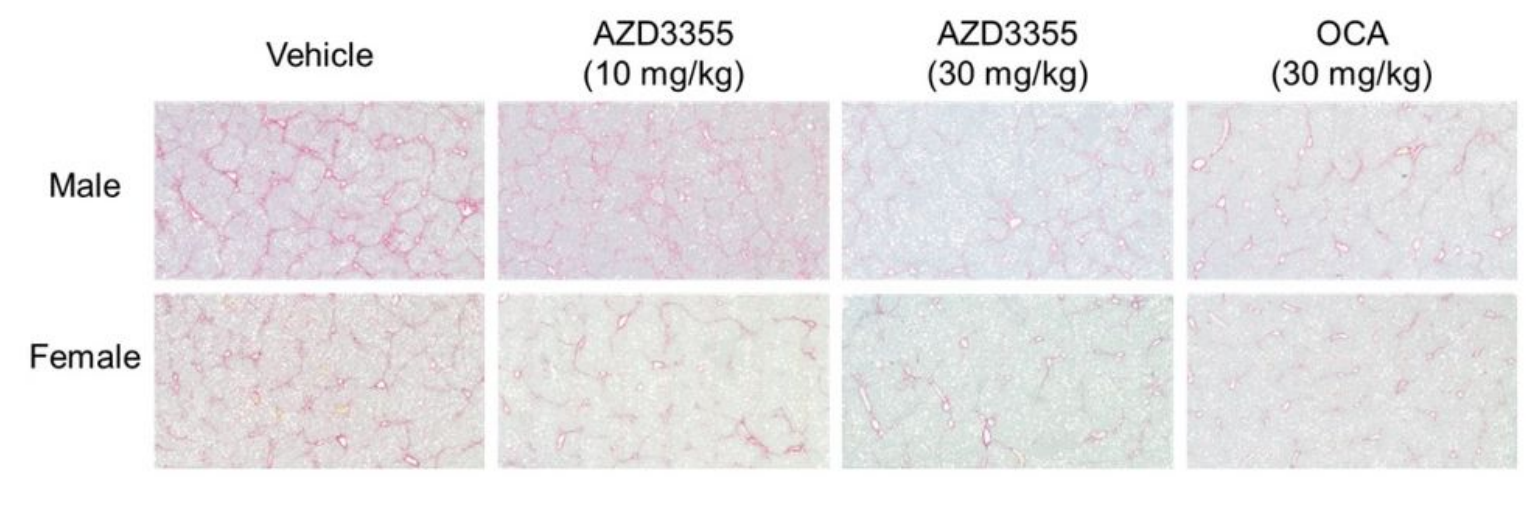

B

Male

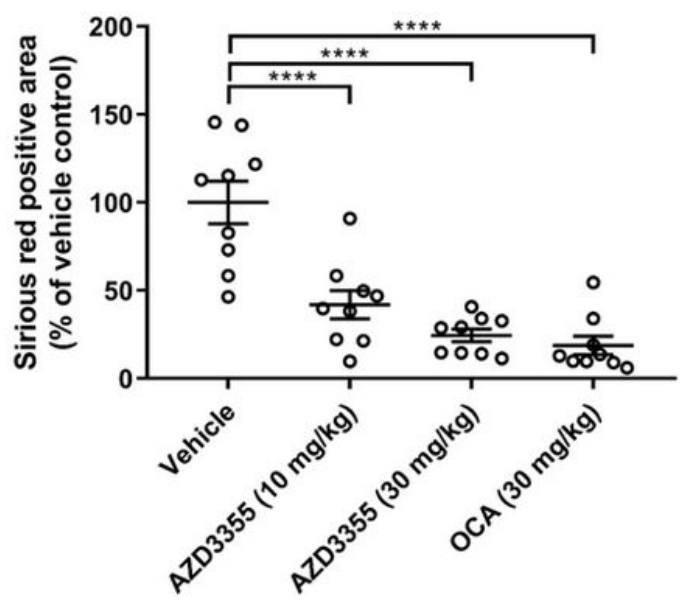

Female

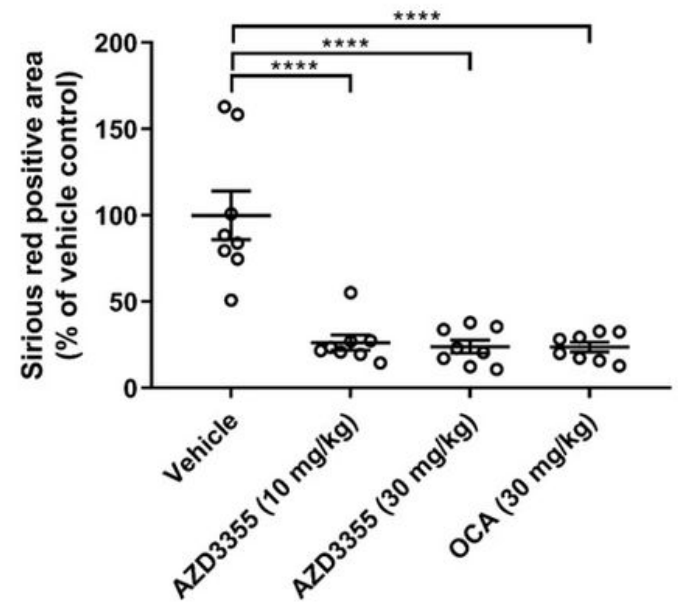

Figure 6

Hepatic collagen deposition is reduced by AZD3355 treatment in a murine NASH model. Representative photomicrographs (20 X magnification) of Sirius Red stained liver sections $(A)$ from male and female mice treated with either vehicle or AZD3355 (10 and $30 \mathrm{mg} / \mathrm{kg}$ ) and OCA (30 mg/kg). Morphometric quantification of Sirius Red-positive area (B) was performed using BIOQUANT image analysis software. Dose depentdent reduction of hepatic fibrillar collagen formation by AZD3355 treatment in both male and female mice was assesed. Vehicle: $\mathrm{n}=9$ male, 8 female; AZD3355 (10 mg/kg): $\mathrm{n}=9$ male, 8 female; AZD3355 (30 mg/kg): n = 9male, 8female; OCA (30 mg $/ \mathrm{kg}): \mathrm{n}=9$ male, 8 female animals. Results were expressed as means $\otimes$ SEM. ${ }^{*} p<0.05,{ }^{* *} p<0.01,{ }^{* \star *} p<0.001$ 
A

Tumor number (Male)

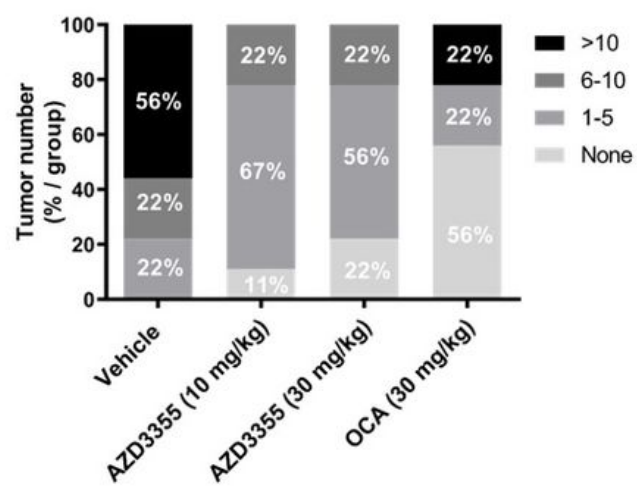

B

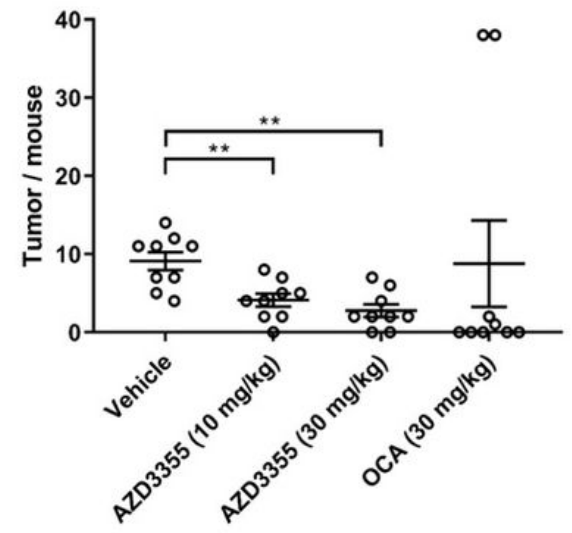

Tumor number (Female)
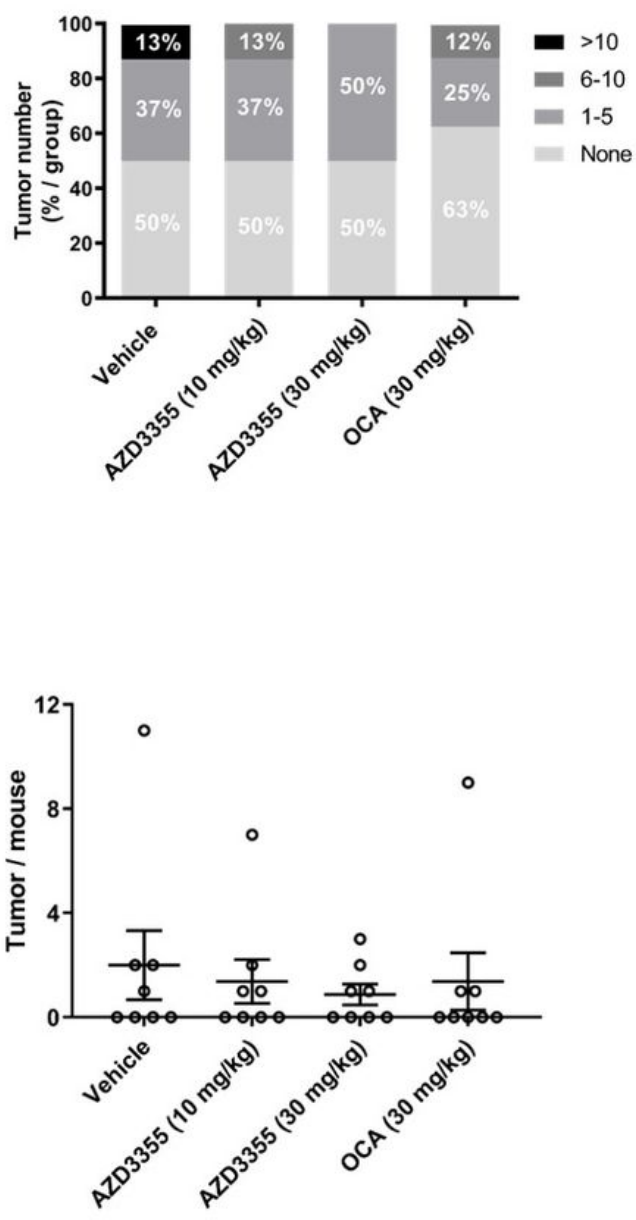

\section{Figure 7}

Reduced tumor development by AZD3355 treatment in murine NASH. Reduced tumor development in male and female mice was evident in AZD3355-treated mice compared to NASH mice treated with either vehicle or obeticholic acid (OCA) (30 $\mathrm{mg} / \mathrm{kg}$ ). As expected, tumor numbers at 24 weeks were greater in males than females in NASH mice treated with vehicle ( $100 \%$ in male and $50 \%$ in female), but both were reduced by AZD3355 treatment (A). Dose dependent reduction of total number of HCC per mouse by AZD3355 treatment was established (B). Vehicle: $\mathrm{n}=9$ male, 8 female; AZD3355 (10 mg/kg): $\mathrm{n}=9$ male, 8 female; AZD3355 (30 mg/kg): $\mathrm{n}=9$ male, 8 female; OCA (30 mg/kg): $\mathrm{n}=9$ male, 8 female animals. Results were expressed as means $\otimes$ SEM. ${ }^{\star} p<0.05,{ }^{\star \star} p<0.01,{ }^{\star \star \star} p<0.001$.

\section{Supplementary Files}

This is a list of supplementary files associated with this preprint. Click to download. 
- Bhattacharyaet.al.SupplementaryScientificReportsApril22021.pdf

Page 25/25 